NIST Special Publication 1219

\title{
Future Generation Wireless Research and Development Gaps \\ Report
}

\author{
Nada Golmie \\ David Cypher \\ Marc Leh \\ Darcy Ziegler
}

This publication is available free of charge from: https://doi.org/10.6028/NIST.SP.1219

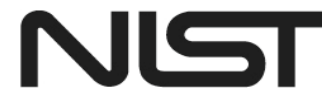

National Institute of Standards and Technology U.S. Department of Commerce 
NIST Special Publication 1219

\title{
Future Generation Wireless Research and Development Gaps Report
}

\author{
Nada Golmie \\ David Cypher \\ Communications Technology Laboratory
}

Marc Leh

Darcy Ziegler

Corner Alliance

This publication is available free of charge from:

https://doi.org/10.6028/NIST.SP.1219

February 2018

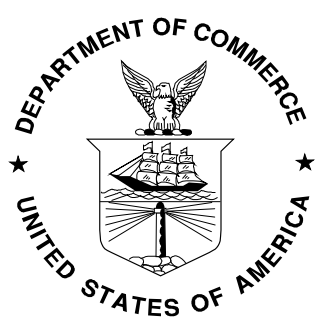

U.S. Department of Commerce Wilbur L. Ross, Jr., Secretary

National Institute of Standards and Technology Walter Copan, NIST Director and Under Secretary of Commerce for Standards and Technology 
Certain commercial entities, equipment, or materials may be identified in this document in order to describe an experimental procedure or concept adequately. Such identification is not intended to imply recommendation or endorsement by the National Institute of Standards and Technology, nor is it intended to imply that the entities, materials, or equipment are necessarily the best available for the purpose.

National Institute of Standards and Technology Special Publication 1219

Natl. Inst. Stand. Technol. Spec. Publ. 1219, 51 pages (February 2018)

CODEN: NSPUE2

This publication is available free of charge from: https://doi.org/10.6028/NIST.SP.1219 


\begin{abstract}
This report identifies and describes several of the most significant gaps within various components of the wireless industry. This report is intended to serve solely as a knowledge product that recognizes the major challenges facing the broader research community, and does not prescribe to make research and development $(R \& D)$ recommendations in light of specific organizations, initiatives, or standards development efforts. The data presented in this report was gathered through market research, a series of stakeholder interviews, and public working group meetings.
\end{abstract}

\title{
Key words
}

Antenna; gap analysis; measurement; spectrum; waveform; wireless communications. 


\section{Table of Contents}

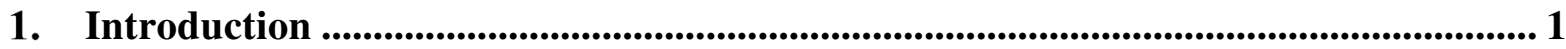

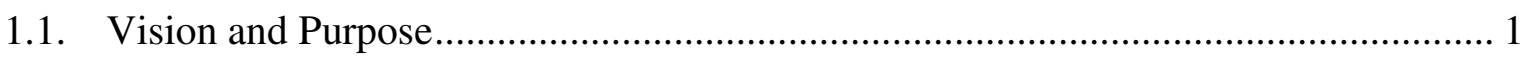

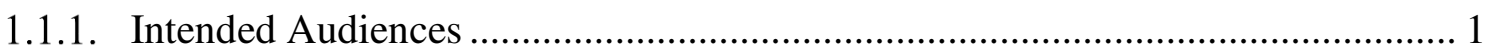

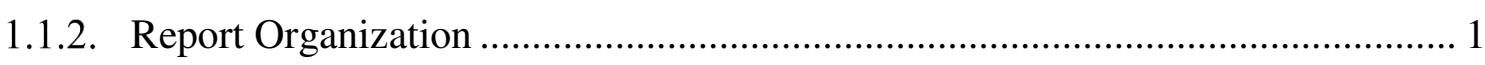

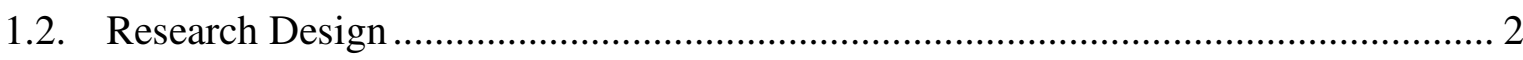

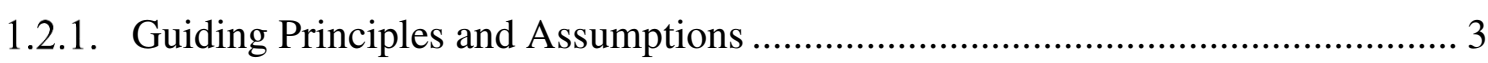

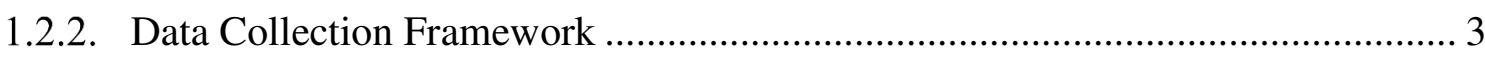

2. Gap Themes Analysis ................................................................................................................ 4

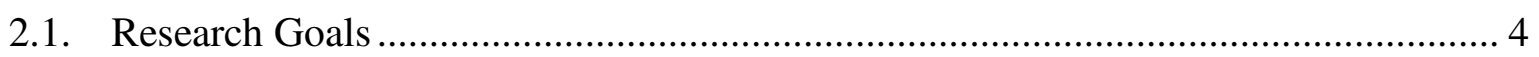

2.1.1. Overcoming the Spectrum Crunch .................................................................. 4

2.1.2. Making Wireless Architectures More Dynamic, Agile, and Innovative ............... 5

2.1.3. Leveraging Analysis and Control Techniques Across Complex Wireless Systems 5

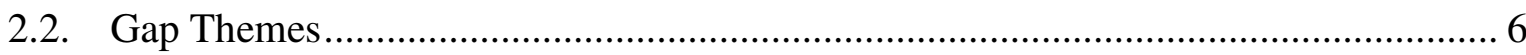

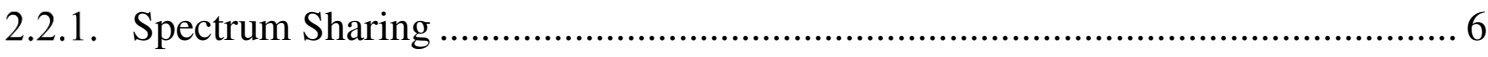

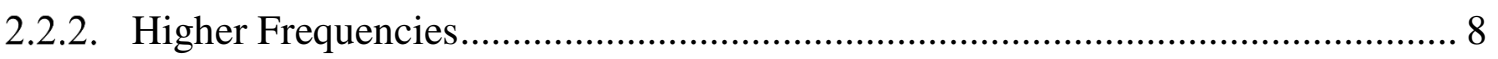

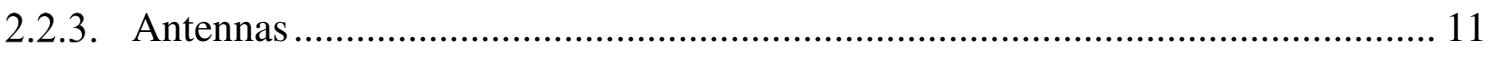

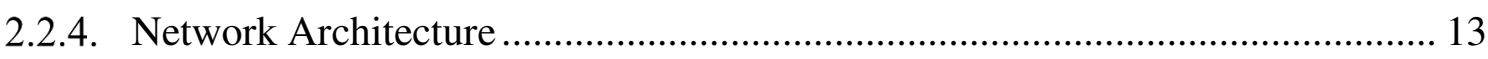

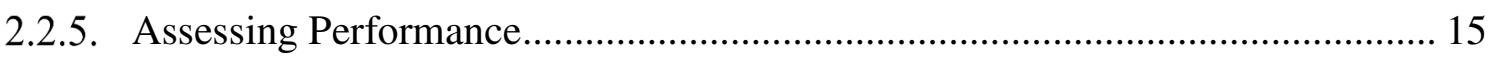

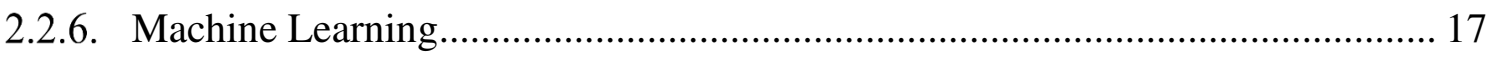

3. Conclusion ............................................................................................................................. 19

Appendix A. Research Methodology ..................................................................................... 20

A.1. Market Research and Stakeholder Interviews (January - July 2016) ....................... 20

A.2. Working Group Meetings (July - December 2016).................................................. 20

A.3. Working Group Roster ……………………………......................................... 22

Appendix B. Appendix B: Full Gaps List................................................................ 24

B.1. Waveform (August 24, 2016) ......................................................................... 24

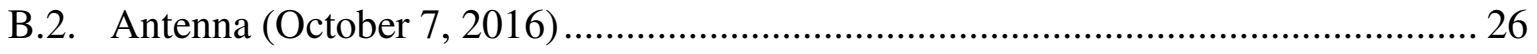

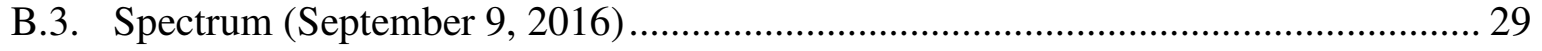

B.4. New Bands (September 22, 2016) .................................................................... 34

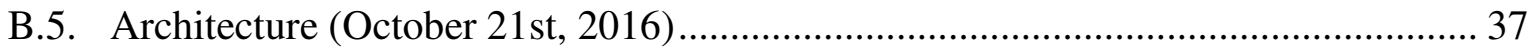

B.6. Protocols (November 4, 2016) .............................................................................. 41 


\section{List of Tables}

Table 1. Working group Roster ................................................................................ 22

\section{List of Figures}

Fig. 1. 2016 Report Development Timeline ...................................................................... 3

Fig. 2. R\&D Gaps Analysis Framework ........................................................................ 4

Fig. 3. 2016 Report Development Timeline ................................................................. 20

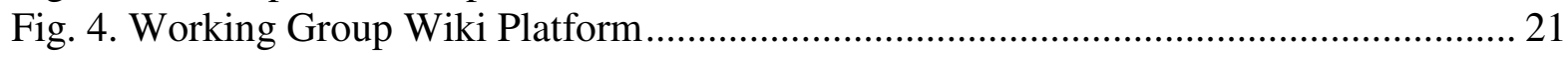

Fig. 5. R\&D Gaps Analysis Framework .......................................................................... 22 


\section{Introduction}

As communications technology evolves beyond $4^{\text {th }}$ generation wireless systems, and as the $5^{\text {th }}$ generation wireless systems $(5 \mathrm{G})$ becomes the industry standard, there is an opportunity to identify the technical gaps and challenges facing the wireless communication industry, over the next 20 years, beyond 5G. Leveraging the expertise represented across its stakeholder base, the National Institute of Standards and Technology (NIST) engaged with organizations across all levels of the telecommunications industry (e.g., wireless carriers, equipment and infrastructure manufacturers, and providers of applications and services). These stakeholders provided valuable input on which measurement, technology, and management challenges need to be addressed.

\subsection{Vision and Purpose}

NIST believes this document will provide valuable guidance to its internal strategic planning as well as serve as a reference for NIST stakeholders in their own planning efforts. This document is not intended to represent a comprehensive overview of every research gap or challenge that needs to be addressed before next generation capabilities are introduced to the marketplace. Similarly, this report is not looking to develop or define a comprehensive research and development (R\&D) agenda. Rather, this report attempts to identify and describe several of the most significant gaps within various components of the wireless industry. This report is intended to serve solely as a knowledge product that recognizes the major challenges facing the broader research community and does not prescribe to make R\&D recommendations considering specific organizations, initiatives, or standards development efforts. The data presented in this report were gathered through market research, a series of stakeholder interviews, and public working group meetings. As a result, these findings reflect the backgrounds and areas of expertise represented in the working group and the information gathered during NIST's market research in Spring 2016.

\subsubsection{Intended Audiences}

This report is intended to identify critical-path gaps that are of interest to a diverse audience. Although NIST sponsored this activity and coordinated the involvement of participating stakeholder organizations, this report is intended to provide value to organizations and researchers across a large segment of the telecommunications industry. The gaps and challenges outlined in this report are too broad for a single federal or commercial entity to address alone.

\subsubsection{Report Organization}

This report is organized into several themes so that readers can focus on specific sections that align with their research needs or interest. The Research Design section provides an overview on how NIST approached this effort and the various data gathering mechanisms and tools used during the creation of this report. A full description of the Research Methodology and working group roster is available in Appendix A.

The Gap Themes Analysis in Sec. 2 identifies the primary challenge areas that the working group identified over the course of Fall 2016. Several topics are described within the six themes included in this section. Each gap theme will touch on the following points: 
- A description of the gap area and why this problem is important for industry to address;

- Specific gaps (highlighted as blue sub-headers) that need to be addressed within this topic area;

- Whether the proposed options for addressing this gap are clear or ambiguous; and

- Examples of enabling actions and actors who are working towards addressing this gap.

Although this report does identify a small number of organizations or initiatives working in the six gap areas, NIST fully understands that this list is not comprehensive of the full scope of activities currently underway. In identifying these enabling actions and actors, NIST is in no way trying to endorse some activities over others. Rather these examples are intended to provide additional context and visibility into various research priorities within each theme. NIST plans on expanding the list of enabling actions and actors within each gap theme during subsequent stakeholder and agency editorial review processes so that the data and corresponding bibliography reflect more accurately current and ongoing research activities. Appendix B includes the full scope of the data collected by the working group and the data is organized by discussion topic area.

\section{2. $\quad$ Research Design}

The NIST team began evaluating future wireless research challenges, gaps, and opportunities in January 2016 by reviewing existing literature and conducting market research. NIST then conducted a series of one-on-one stakeholder interviews to further inform the market research findings before convening a public working group in August 2016. The following section provides an overview of the framework used to collect input for this report. Please refer to Appendix A for a full description of the data collection process, the purpose of each step in the report development workflow, and methods that were used to collect data from subject matter experts in the wireless industry. The working group roster is also included in Appendix A. 


\section{Timeline}

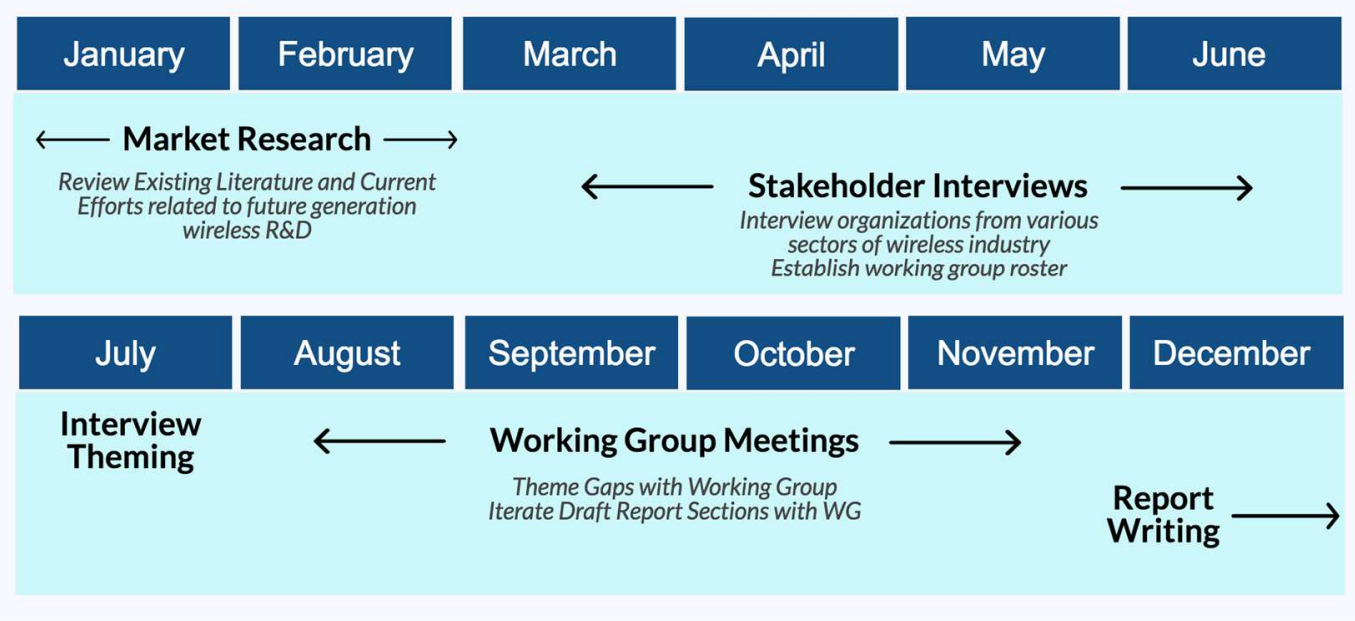

Fig. 1. 2016 Report Development Timeline

\subsubsection{Guiding Principles and Assumptions}

The following principles and assumptions have guided NIST's efforts during the development of the Future Generation Wireless R\&D Gaps Report:

- This effort was not about defining another vision for future generation wireless communications as there are already recently published industrial, academic and government white papers on that topic.

- Different performance requirements and expected capabilities for future generation wireless networks were considered, often as implicit assumptions, to identify the key measurement, technology, and management gaps that need to be addressed to realize these expected wireless capabilities.

- Transparency throughout the data collection process was promoted so that final conclusions could be attributed to specific working group meetings and stakeholder input.

- The report framework and data collected through this framework reflect working group input and are not intended to be comprehensive or representative of the entire wireless industry.

- The reference to technical approaches and projects aiming at addressing some of the gaps identified are for illustrative purposes only. These references were often mentioned during the discussions, they are not exhaustive nor comprehensive and they do not represent the endorsement of any specific activity or effort.

\subsubsection{Data Collection Framework}

NIST's market research and stakeholder engagement revealed several components of the future wireless industry that would benefit from additional focus. Prior to convening the stakeholder working group NIST developed the following data collection framework (shown 
in Fig. 2) to ensure that this report was informed by a sufficiently well-rounded set of responses and inputs. Over the course of 2016, NIST's working group met biweekly to discuss each framework vertical (and associated measurement, technology, and operational gaps) in depth:

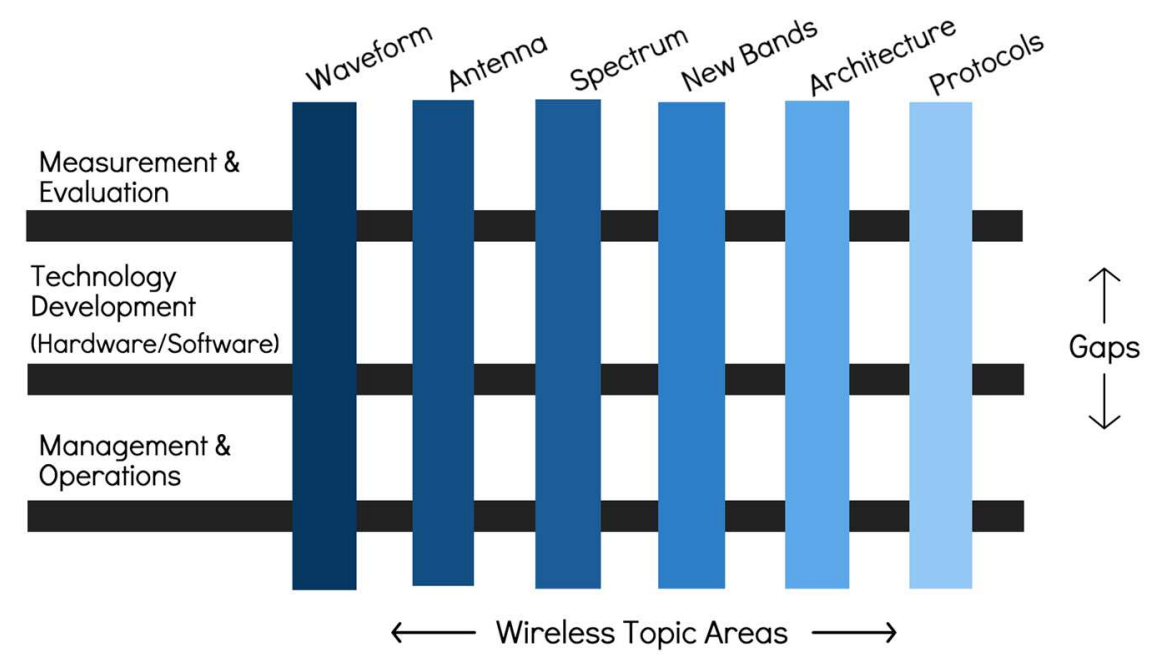

Fig. 2. R\&D Gaps Analysis Framework

\section{Gap Themes Analysis}

During the creation of this report, several themes emerged throughout the one-on-one interviews and working group discussions; these themes are either cross-cutting in nature or foundational to the development and evaluation of wireless communications technologies. To improve readability and ensure the most comprehensive analysis of the data collected, NIST organized the final list of technology gaps by theme rather than by discussion topics. These themes represent the areas of research that were most commonly discussed during the working group process and could be considered the most pressing or important challenges discussed by the working group. This section summarizes the key discussion points within each area of research need. To view the full list of discrete gaps associated with specific working group discussion topics, reference Appendix B.

\subsection{Research Goals}

Before identifying specific technology gaps that need to be addressed prior to the introduction of future generation wireless network capabilities, it is useful to lay out several industry-wide research goals that would be realized by doing so. These research goals, which the working group sometimes referred to when discussing gaps, can be thought of as a set of assumptions as to why the subsequent gaps identified in this report need to be addressed. By dedicating research and development resources to solving the challenges described in the Gap Themes Analysis section of this report, the wireless community will contribute to achieving the following research goals:

\subsubsection{Overcoming the Spectrum Crunch}

The demand for wireless bandwidth has never been higher than it is today, and the rapid proliferation of data-intensive applications and services will only contribute to overloading the frequency bands and spectrum management techniques used today. Recognizing this 
need, the Federal Communications Commission (FCC) unanimously voted to open $10.85 \mathrm{GHz}$ of new spectrum above $24 \mathrm{GHz}$ in July 2016 to facilitate high-capacity, highspeed, and low-latency networks that will support next generation wireless applications. However, releasing additional licensed and unlicensed spectrum to the wireless community will likely not be sufficient in and of itself to solving the spectrum crunch and accommodating the dramatic rise in network demands in the coming years. The working group discussed several measurement and technology challenges that need to be overcome, ranging from improved channel measurements and models, interference assessments and mitigation techniques, to new antenna designs and algorithms to take advantage of higherfrequency bands. In addition, improving spectrum sharing and re-use and creating smaller cells to allow for greater capacity also will play a role in overcoming future stresses on spectrum, but these techniques may require additional backhaul connections or infrastructure which makes using them in isolation a costly alternative to leveraging an ensemble approach (access to new spectrum, improved spectral efficiency, and improved re-use techniques deployed together.) These solutions will need to be paired with more innovative spectrum sharing techniques to ensure that occupied or new spectrum bands are maximized to their full utility, at a reasonable cost, and made available to an increasingly diverse set of wireless consumers.

\subsubsection{Making Wireless Architectures More Dynamic, Agile, and Innovative}

Just as increased demand for higher, more ubiquitous wireless bandwidth and connectivity will require the acquisition of new spectrum and more efficient use of it; new network architectures will need to be developed in order to effectively manage resources, heterogeneous devices and applications, and dynamic performance requirements associated with future generation wireless capabilities. Adaptive network performance management will help support the diversity of applications and services relied on by mobile consumers, public safety, utilities, and non-traditional wireless industries. High-speed networks such as massive broadband or fiber will need to seamlessly communicate with ad-hoc deployable networks to ensure that components such as critical information infrastructure (such as a highperformance data server) and more localized data collection tools (such as a sensor-based network at the edge) work together to create an exceptional end-to-end wireless experience.

\subsubsection{Leveraging Analysis and Control Techniques Across Complex Wireless Systems} Expanded use of data science and machine learning techniques across all components of the wireless network will help optimize resource allocation, provide more detailed visibility into network performance, and even help mitigate interference concerns through, for example, intelligent beamforming and beam steering of next generation antenna technology. Even as computing power available to wireless providers increases and the cost associated with storing data decreases, machine learning algorithms will be deployed to minimize packet transport time, power consumption of wireless sensors or endpoints, and network downtime. In addition to optimizing network traffic, increasing resiliency, and providing more personalized data and services to wireless customers, data science will support more intelligent network infrastructure planning and architecture through enhanced location-based services.

Techniques for analysis and control across complex systems cut across many of the gap themes outlined throughout this R\&D Gaps Report. More sophisticated analytics will also play a key role in overcoming the spectrum crunch and making network architectures more 
agile. By addressing the challenges outlined below, the wireless research community will better position itself to realize these industry-wide research goals.

\subsection{Gap Themes}

\subsubsection{Spectrum Sharing}

Improved spectrum sharing will compliment dedicated spectrum use in the future generation wireless economy to provide higher data rates, more seamless machine-to-machine communications, and innovative network architectures necessary to realizing anticipated performance expectations. It is a key enabler in support of applications and services that demand increased capacity on future wireless networks. Techniques for efficiently sharing existing spectrum have received some attention so far but will require additional R\&D if wireless stakeholders intend to keep pace with accelerating consumer demand. The working group defined the spectrum vertical as "Methods for coexistence of independent systems that use radiating electromagnetic signals; understanding factors in the electromagnetic environment that affect the performance of such systems and their coexistence." Using this definition, working group participants identified gaps related to measurement; technology development; and management and operations that are of the most important to the wireless research community over the next 20 years.

\subsubsection{Spectrum measurements}

Spectrum can be shared geographically, temporally, or between users to increase spectrum access to wireless consumers and providers. Spectrum sharing techniques rely on spectrum occupancy measurements that are typically used to determine whether new transmitters can be added without causing additional interference. However, assessing interference remains challenging. Spectrum measurements should differentiate between the various communication and non-communication sources of interference, including intentional radiators, man-made noise, natural noise, and intermodulation spurs. The working group consistently discussed the need for improved models, tools, and metrics to assess and mitigate interference, and the ability to emulate and simulate realistic interference environments.

Another significant challenge in characterizing the overall radio frequency $(\mathrm{RF})$ environment is the proliferation of highly directional antennas so the idea of having a measuring device that simply listens to all the emitters "around" it no longer works. Spectrum monitoring in such a directional environment becomes significantly more challenging since interference needs to be measured with respect to each receiver. Accurate spectrum monitoring will be hampered in this environment as the spectrum monitoring sensors may not detect wireless activity in the cases where the directional antennas are steered away from the sensing system. Similarly, having spectrum measuring centers where highly sophisticated and calibrated systems are used to report on everything that the system detects will be mainly applicable to the case where the victim receiver is fixed. Capturing a well-rounded, coherent characterization of the spectrum may require monitoring to occur in more than one place.

Given the directional propagation properties of higher frequencies, interactions between devices in the millimeter-wave band will be highly local. As a result, real world measurement will be performed by the devices themselves and their neighboring devices, creating modest data flows from many uncalibrated devices that need to make sense of noise in the process. Specifically, the working group indicated that there is a need to gain a better understanding 
of what spectrum is being used or underutilized by developing new approaches to spectrum monitoring such as developing devices that could self-report what they are experiencing (including standards for self-reporting data and calibration for devices doing the selfreporting), and discovering new methods for verifying which bands these devices are using when self-reporting their experiences. These new approaches are likely to significantly change the market strategies of key telecommunications industry actors.

Location databases where interference predictions are computed in advance and rely on accurate propagation models, may not be sufficient as in the case of free space optical, and higher-frequency bands. Other approaches need to be developed where interference is predicted on the fly after systems are deployed and powered up.

\subsubsection{Spectrum sharing techniques}

Better components, devices, techniques, and algorithms are needed to effectively manage interference between various systems operating in the same bands. More specifically, there is a need to develop inexpensive, small, higher-quality RF filters that are frequency tunable so that more users are fit into the spectrum without the added and unnecessary complexity elsewhere in the system. High-power transmit filters are potentially more challenging than receiver filters. While good signal-processing techniques are available today, it remains difficult to implement software-defined filters. In addition, more sophisticated transmission power management techniques are also needed to improve spectrum sharing.

System-level approaches including collaborative, non-collaborative approaches need to be developed. When collaboration channels are present, industry needs to determine the information that should be shared among networks to facilitate efficient coexistence. Currently, the Defense Advanced Research Projects Agency (DARPA) Spectrum Collaboration (SC2) program may be developing solutions in this space. Another example is the discussion in Institute of Electrical and Electronics Engineers (IEEE) 802.11ax task group considering local vs. global optimization in mitigating interference.

A promising path forward for improving spectrum sharing is a greater use of adaptive algorithms to more efficiently and automatically manage spectrum sharing. Adaptive spectrum sharing systems will need to operate within very clear rules stating how to collaborate with new radio systems, share spectrum across systems, and optimize resource utilization. These analytics would enable automated spectrum monitoring and enforcement, making networks more resilient to jamming and responsive to security threats. However, the rules guiding machine learning on wireless systems have not yet been defined. This and the significant computational requirements associated with wireless machine learning deployments are key barriers preventing more effective, automated spectrum sharing.

There is a need to develop more integrated spectrum situational awareness, command, and control architecture to enable controllable, adaptive, and flexible operations of hardened spectrum dependent systems.

\subsubsection{Spectrum sharing evaluation: testbeds \& metrics}

There are currently a number of testbeds that are in use (https://www.nitrd.gov/apps/wsrdtestbedinventory/). However, due to technical and cost considerations they tend to be specialized to particular radio and radar technologies. Thus there is a need to develop more general, large-scale testbeds that reflect more realistic 
scenarios to evaluate coexistence performance. A few projects and initiatives including the National Science Foundation's (NSF) Platforms for Advanced Wireless Research (PAWR), the FCC program experimental license and the DARPA spectrum collaborative challenge may have begun to answer this need.

In addition to testbeds, evaluating spectrum sharing requires the use of metrics established to qualify the effectiveness or the so-called coexistence of different systems utilizing the same spectrum. Sharing is more difficult to achieve across heterogeneous or different types of systems and priorities, and the sharing metrics need to account for multiple sharing dimensions, encompassing the system performance across all layers of the networking stack. More specifically, the metrics need to account for what agents operate in a given spectrum band, what types of information exchange they care about, and what priority access they have. While some metrics are technology agnostic and consider overall spectrum efficiency and utilization, there needs to be different metrics that more accurately characterize the sharing as "seen" or experienced by different systems. Metrics considering group versus individual optimization should be considered in addition to the overall throughput and opportunity cost. These evaluation metrics together with realistic testbed environments should encourage the evolution towards better spectrum sharing technologies.

\subsubsection{Spectrum management}

Traditional spectrum management focuses on coverage optimization for noise limited systems with minimal frequency reuse. In millimeter-wave bands where propagation is greatly affected by small changes in device location, spectrum management gets much more difficult. In addition, a dense deployment for higher bands is needed to provide a sufficient probability of service in highly challenging propagation environment. Improved methods of providing reliable service in a shared-spectrum environment are lacking. The working group identified the potential for a new spectrum management challenge associated with supporting continuous coverage in this new environment.

Participants recognized the need to develop a consensus or shared vision of how the spectrum is managed, what types of control (centralized versus distributed), and operation modes (licensed versus unlicensed) are used. Adequate rules for resolving conflicts resulting from sharing in all of these regimes and operation modes are needed. Thus designing appropriate spectrum access systems for both outdoor and indoor operation covering both licensed and unlicensed bands up to $100 \mathrm{GHz}$ are needed. Commercial systems today are able to detect, characterize and report system performance impacts due to RF energy incoming from independent spectrum users. This is done via key performance indicators (KPI). However, KPIs are not designed to indicate the source of interference, only its effect on performance. Having this capability leads to an electromagnetic spectrum dynamic planning, directing, and control capability to conduct real-time spectrum operations in heterogeneous electromagnetic environments.

\subsubsection{Higher Frequencies}

Given the exponential increases in wireless demand, future generation networks will need to leverage higher frequencies in conjunction with existing technologies operating below $6 \mathrm{GHz}$ in order to meet the performance expectations of seamless user connectivity, improved speed, and ultra-reliability. Expanded use of millimeter-wave (mmWave) frequencies, or frequency bands between $28 \mathrm{GHz}$ to $300 \mathrm{GHz}$, represents a commonly cited enabling technology that 
could accommodate the increased network capacity, bandwidth, and connectivity needed to support the billions of new users and devices that will rely on wireless communications in all aspects of everyday life in the near future.

The wide range of spectrum (above $6 \mathrm{GHz}$ and up to THz) expected to help meet these demands led the working group to describe this topic area more generally as New Bands - or methods for exploiting previously unused or lightly used portions of the electromagnetic spectrum - rather than focusing solely on the mmWave radio band frequencies. Although there was no consensus among the working group on the desired frequency range that industry would like to see supported, utilized and measured, the group agreed that moving to higher frequencies than typically used today, presents a unique set of challenges. These challenges include many unknowns related to signal propagation and its properties, lack of adequate design tools and metrology to develop new waveform designs, modulation schemes, and filtering and signal processing techniques. In addition, the research community will need to gain an improved understanding of how these new bands behave in a variety of emerging usage scenarios, how to seamlessly operate radios across existing and new bands, and how to combine new bands with advances in antenna technologies. These areas represent a small fraction of the measurement, technology development, and operational gaps that need to be addressed before new bands can deliver on the promise of increased wireless capacity, mobility, and connectivity.

The remainder of the section provides an overview of the specific gaps that need to be addressed in order to overcome the challenges associated with higher frequencies.

\subsubsection{Physical propagation characteristics}

Researchers looking to increase network capacity through expanded use of new, higherfrequency bands experience propagation and system modeling challenges simply because there is still a lot to be learned about how signal propagates through these new bands for future generation use cases. Accurately characterizing propagation in higher-frequency bands in various environments is a critical first step in designing future generation systems that take advantage of spectrum in the higher bands.

The lack of comparable measurement data for live systems and mobile environments across a large range of frequencies $(>60 \mathrm{GHz})$ is a key challenge limiting industry's ability to design, develop, and verify the performance of next-generation wireless systems and hardware. Organizations conducting measurement campaigns in high frequency usage scenarios usually do so with disparate methodologies, measurement devices, and environmental parameters; making it difficult to compare results and therefore extract measurement data to develop sufficiently detailed channel models over a large frequency range. Without more comparable measurement data that quantify the propagation effects of higher frequencies on specific mobile applications and common practices for translating measurement campaign results in channel models, wireless standards development and hardware optimization efforts will stagnate.

In order to accurately evaluate the performance of future generation network environments via channel models, researchers will need to understand how new, higher-frequency transmissions will interact with the rest of the system. Therefore, there is a specific need for greater measurement characterization of widely deployed multi-beam antenna arrays before 
accurate channel models can be developed. Future network environments will also require multiple measurement devices that are optimized for different purposes (i.e., mobile device vs. a grounded, calibrated device) and an architecture that can handle the variety of these devices. In addition to advancing the underlying measurement science required to understand this new architecture, industry will need to develop stronger metadata standards that describe not only the measurement data, but how the measurement was taken, the positioning of the transmitter and receiver at the time of measurement, and other channel sounder and environmental parameters. Improved characterization of the propagation of higher frequencies will lead to much more innovative and efficient wireless system design and planning.

Several international research consortia currently conduct measurement campaigns in a widerange of commercially interesting frequency bands. While frequencies above $60 \mathrm{GHz}$ do not receive as much research attention as the recently FCC-licensed $28 \mathrm{GHz}, 37 \mathrm{GHz}$, and $39 \mathrm{GHz}$ bands; organizations such as Mobile and wireless communications Enablers for Twenty-twenty (2020) Information Society (METIS), Millimeter-Wave Based Mobile Radio Access Network for Fifth Generation Integrated Communications (mmMagic), IEEE, Third Generation Partnership Project (3GPP), European Cooperation in Science and Technology (COST), and the 5G mmWave Channel Model Alliance support researchers who are focused on conducting measurements at these higher frequencies.

\subsubsection{System components: antennas, circuits, and waveforms}

As the number of antenna elements increases with the use of higher frequencies, there is a need to study what is the optimal massive antenna array architecture with limited RF chains and power consumption. There is also a need to research how to efficiently integrate the antennas with the RF circuits. This technology gap is receiving a lot of attention in academia and industry today. For example, Massive Multiple Input Multiple Output (MIMO) technologies are the subject of many publications and demonstrations today. Current challenges related to this gap arise in multiple domains such as circuitry, signal processing, power dissipation, and impractically large physical size (distance between antennas needs to be relatively large in relation to wavelength). The solutions in this space may be very promising, but it is not well known when they will be deployed or how practical they will be.

As already discussed in the previous section on spectrum sharing, managing interference at higher frequencies will require the development of receiver circuits and filters that can reconfigure themselves to remove intermodulation out of the passband.

There is also a need to develop cost-effective steerable antennas for mobile devices and the algorithms to control them at both ends of the link to overcome dynamic 3D fading and blocking. Today we can only create 2D spatial fields that work well at low frequency, but at mmWave frequencies the ability to control the wave fields is a function of angular relationship of antennas.

In order to get the link budgets working well, steering is required at both ends of the link. It is more difficult for a mobile device to implement steering. Thus, a greater deal of agility in mobile device antenna design is needed to match that of a base station.

Similarly, new waveforms need to be developed for higher frequencies, in addition to advanced tools, models, testbeds, and fundamental mathematical techniques to analyze these 
new waveforms will be needed as well. This will allow the community to understand how well each waveform facilitates efficient and effective use of the spectrum, in addition to deriving any fundamental limits that exist for new waveforms.

\subsubsection{Range extension}

So far, given the limited range of mmWave frequencies and other higher frequencies, their use has been primarily limited to short-range indoor communications such as personal area networks, server rack communications, and other high-density machine-to-machine networks. A challenge identified by the working group is the need to extend the range achieved with higher-frequency bands so that they can be used for a wider set of applications.

Atmospheric or environmental attenuation, blockage and path loss cause rapid loss of signal strength when transmitting at higher frequencies. The signal propagates in primarily line-ofsight conditions which limits its transmission range to hundreds of meters even in scenarios that lack the buildings, foliage or environmental conditions that normally would block, fade or attenuate the signal. These attenuation realities become especially challenging in instances of rain, foliage, or reflective surfaces. One technique to overcome the greater propagation loss experienced at higher frequencies is to use directional antennas, or antenna arrays, which can provide increased gain in the direction that the transmission is steered, and almost no gain in all other directions. This forces systems that use highly steerable antennas to sacrifice widespread coverage in favor of greater capacity and throughput in a single direction. However, there are many challenges associated with the use of directional antennas at higher frequencies including new circuit designs, beam-training procedures, and beam-training algorithms that need to be developed to assist in controlling the various antenna elements to more precisely steer beams to desired receiver locations.

Deploying innovative architectures such as massive MIMO and ultra-dense heterogeneous networks within mmWave band represents another technique for overcoming the limited range and allowing the use of mmWave frequencies outdoors. Future generation devices may also be able to more accurately recognize and process signals at higher frequencies from longer distances, but commercial solutions to do so have not yet been developed.

Finally, pairing high frequency networks with cellular and/or satellite networks operating in different bands may yield greater coverage while maintaining the increased capacity and throughput enabled by higher frequencies. However, when integrating higher-frequency systems with the wider-area networks mentioned above, signal coexistence and managing interference becomes a significant challenge. The working group identified the coexistence of $28 \mathrm{GHz}$ and satellite systems (which uses $27.5 \mathrm{GHz}$ to $30 \mathrm{GHz}$ and $17.3 \mathrm{GHz}$ to $21 \mathrm{GHz}$ bands for uplink and downlink respectively) as a scenario currently receiving attention from the research community to overcome this coexistence challenge.

\subsubsection{Antennas}

While significant progress has been made in recent years in deploying innovative antenna designs such as adaptive arrays; multi-beam MIMO; and reconfigurable antennas in terms of radiation pattern, gain, or frequency response; several specific technology and verification gaps need to be addressed before industry can truly realize a capacity- and capabilitymaximized wireless system. When identifying these gaps, the working group defined the 
Antenna vertical as "Methods for transducing information between radiating electromagnetic signals and wired representations suitable for generation or detection."

\subsubsection{Improved design}

Developing new antennas is difficult and costly, and the working group cited the ability to rapidly develop cost-effective, power-efficient mobile antenna arrays represents a significant engineering gap going forward.

More specifically, the working group noted that the need for improved antennas with higher gain over broader frequency ranges has been a persistent challenge for wireless researchers over the past 20 years. The working group argued that there is a need for antennas that can support simultaneous signal transmission and reception over a very wide frequency band (500 MHz to $100 \mathrm{GHz}$ was the range specifically cited by the working group). Maintaining consistent array performance over this wider frequency band makes embedding next generation antenna arrays in traditional mobile devices difficult from a form-factor perspective because the vastly different wavelengths represented in the wider frequency band require the distance between antenna elements increase as well. As a rule of thumb, the distance between antennas is at least one wavelength; for frequencies above $2 \mathrm{GHz}$, the antennas can be $12.7 \mathrm{~cm}$ (5 inches) apart or less, and for millimeter-wave bands, the distance is small enough to fit into a phone. Discovering an optimal antenna array architecture that can effectively process outgoing and incoming signals over a broad frequency range within a reasonable form- and cost-factor will be essential to widespread commercial adoption of technologies such as Massive MIMO and ultra-wideband antennas and their integration into traditional radio frequency circuits.

Also, as higher frequencies are utilized for wireless communications, the number of antenna elements will increase, but the number of RF chains will remain limited due to power consumption concerns. Studying how to integrate different antennas for different bands in a practical mobile device will become more important over time as wireless systems and applications increasingly rely on interoperable low, medium, and high band (mmWave) frequency capabilities. Current challenges related to this gap arise in multiple domains such as circuitry, signal processing, power dissipation, and impractically large physical size of the device.

The working group posited that next generation antennas will have variable radiation parameters, such as beam width, radiation patterns, and directionality. Antenna designs will need to use these variations (in terms of beam width and beam steering) in order to maximize network capacity and reduce interference. Over time, the working group expects antenna performance metrics such as rate of adaptation, beam widths, gain-to-rejection ratios to be tightened as capacity demands on future generation networks increase. "Capacity Maximized" was defined by the working group as the ability to build antennas with tunable frequency nulls that result in more capacity. In spectrum sharing environments, transmitter nulls will be relied upon heavily to reduce interference at receivers in fixed locations. However, developing an optimal nulling strategy remains difficult and is limited by the tools available to characterize and assess interference. New antenna designs that have rapidlytunable frequency and spatial response "notch filters" have been developed for military applications. However, these electrically tunable and steerable antenna designs are not incorporated into today's deployments. 


\subsubsection{Testing and validation}

As antenna systems become more complex over time they will require even more complex measurement and validation setups. Today's state-of-the art antenna performance testing methods will need to move beyond managing gain and signal recognition.

A major challenge cited by the working group is that conditions in an anechoic chamber do not reflect what they would be in the field. Antennas in the field, especially ones used in handheld devices are highly affected by the environment. Measurements performed in an anechoic environment do not have an electromagnetic environment similar to that found in a dense urban environment. Test setups are needed to accurately and reliably emulate what happens in the field, including an accurate assessment of multipath effects, for different environments, mounting scenarios, and interference levels. The FCC tests various devices by emulating the conditions that the product will be placed in when sold. However, these conditions are not always entirely accurate for real world deployment. Thus, industry needs new ways to simulate interference for different deployment environments. Additionally, there is a need to develop standards for the antenna's context of use and when to test antennas in a field environment versus a laboratory environment. The working group did point out that while standards exist for antenna performance today, they do not exist for the architecture of the antenna and they do not specify what industry needs to use.

More specifically, there is a need for standardized interfaces and connections for automated tests of mmWave antennas and performance. Testing of mmWave antennas in the future will have to be radiated with the most important tests being those for interference cancellation, signal acquisition and tracking in a dynamic 3D environment including body shadowing and blocking effects - none of which are available today. Although 3GPP currently is working to resolve the performance testing gaps associated with mmWave antennas, a strong goldstandard for standardized methods for specifying performance requirements and testing these performances would benefit industry well into the future.

\subsubsection{Network Architecture}

The working group defined the concept of emerging network architectures as "Methods for designing systems that effectively exploit Waveform, Antenna, Spectrum and Protocol technologies to provide communications services." One of the primary research gaps related to new network architecture design is that current wireless architecture will not be able to sustain the increasing diversity of devices and applications over various networks at reasonable development and operational cost if industry solely relies on incremental improvements. Wireless network architecture design will need to break from traditional models and realize true innovation to build sufficiently secure, scalable wireless systems that can accommodate the increased capacity and reliability demands of future generation applications. The following gaps were identified by the working group as needing to be addressed to facilitate the fundamental innovation of architecture design necessary to accommodate the impending capacity, security, and management pressures that ubiquitous application, device, and user connectivity will put on future generation networks.

\subsubsection{Heterogeneity, density and agility}

Future generation network architectures will need to feature more flexible access technologies that can accommodate new internet of things (IoT) devices with disparate operating systems. Network design, architecture, policies, protocols, and antennas will need 
to become increasingly dynamic rather than static in order to manage resources efficiently in this new system. Extending current architecture models - those that rely on cell towers, fixed dishes, and macrocell nodes - will not be enough to accommodate the exponential increase in connected applications, data flows, and devices. Augmenting the cell tower or fixed dish model with expanded use of technologies such as deployable networks, mobile hotspots, and massive MIMO antenna arrays will lead to increased network capacity and coverage.

However, these supporting technologies need to be designed with respect to cost, scalability, spectrum, and form-factor requirements that have yet to be defined.

Ultra-dense networking was also identified as an area in need of additional research. Future generation networks will be exponentially more complex, and mobile devices will need to prioritize certain spectrum bands and base stations over others when they are moving within the coverage range of multiple options. Devices and base stations currently lack the ability to autonomously self-organize and prioritize network traffic; more research needs to be done to find better techniques for coordinating communication between network layers in response to device mobility, data transmission, and spectral availability and efficiency. In order to meet future generation wireless performance requirements such as higher speeds, lower latency, and flexible integration of software-defined network components; the wireless industry will need to develop standardized architectures and test specifications for fundamentally new system design.

\subsubsection{Local processing}

The ability to decentralize network capacity to a distributed set of wireless nodes will enable faster and more scalable communications by regionalizing, or layering, components of the core network and reducing backhaul requirements. Increased reliance on mesh networks, deployables and small cell networks will minimize latency and therefore support more reliable device-to-device communications. Several working group participants described this shift towards decentralized architectures as a "fog network" rather than consolidating network components in a centralized core. The demand for increased bandwidth and reduced latency puts pressure on cloud-based networks to push computing capabilities closer to the user, which will necessitate researchers to overcome a cognitive barrier to shift from traditional fixed tower architectures (one-to-one) to architectures with a greater number of deployable components (one-to-many).

Furthermore, there is a need to develop software mechanisms that enable effective operation of pieces of a system when unexpected conditions arise in the environment or in the system itself; or when connectivity to the core network is impaired. Some working group participants argued that the notion of "core networks" will only serve to provide broad guidance to distributed network components closer to the edge user related to how to collect, process, and transmit information. Regardless, the reliability of these decentralized networks will be severely tested as mission-critical applications and services use multiple network layers or nodes to transmit information (meaning that a single point of failure may compromise the entire system). The resiliency of distributed network architectures will rely on complex software-based mechanisms that govern how networks operate when new users or devices come online or offline; and the working group noted that there is little current research focused on these mechanisms today. 
While the working group noted that a fiber optic infrastructure will deliver much greater bandwidth closer to the user, at some point all network architectures will be expected to make "the last hop" wirelessly. Today's research community has not articulated how to design or manage this distributed architecture, specifically how an organization would maintain the thousands or millions of distributed server racks in dense urban environments that are expected to support future generation wireless application demands. Data communication protocols will also need to be designed in order to limit backhaul requirements.

\subsubsection{Light control}

The working group argued that there is a need to fundamentally rethink and redesign the control plane to improve network performance such as control plane latency data path throughput, and scalability. Current protocols for wireless (both cellular and to a lesser extent $\mathrm{WiFi}$ ) are too heavy-weight and are borne out of a reliance on a circuit-switch legacy designs. The weight of carrying legacy architecture designs in future designs will restrict how well industry can support future applications and evolve wireless architecture in terms of improved data traffic (for example, supporting IoT applications) or redesigning network topology (for example, the proliferation of small cells described earlier). Today, mobility is handled inefficiently. Specifically, the control protocol in the cellular context maintains excessive amounts of session state data, and involves a very large number of message exchanges to establish a communication session, handle mobility or deal with other mobile device state transitions. Because future generation network architectures may partition functions across a number of distributed components to a greater degree than what industry uses today, control plane protocols may also present significant inefficiencies. Nextgeneration control plane protocols will need to be designed to improve network flexibility and scalability.

One potential path forward to addressing the inefficiencies related to legacy circuit-switch protocols may be to re-partition how these protocols are implemented by separating the control plane and the data plane. Today, there is minimal effort being spent in re-thinking how these protocols should be put together. Even WiFi networks represent a certain amount of complexity with triangular routing to deal with mobility, and current research efforts related to solving mobility issues are not sufficient. In order to prepare for an increasingly dynamic networking landscape, some researchers argue in favor of fitting all network layers into the notion of software-defined network. This idea holds appeal because wireless control plane interactions with the data plane are highly granular and require consistency.

\subsubsection{Assessing Performance}

Next generation wireless technology promises to introduce significant advances in latency, bandwidth, throughput, error rate, and a variety of other network performance metrics. However, given how the wireless environment is expected to change in the coming years moving towards a decentralized architecture, increased reliance on higher frequencies, and a heightened demand for ubiquitous data - researchers will need to innovate the ways in which they measure and model network performance to accommodate these new factors.

For example, there is a significant need for new mathematical and simulation models to predict network performance, and the need for improved metrics of assessing the accuracy and reliability of network performance. The research community will also need to develop 
new algorithms to improve network performance. Lastly, wireless providers will need to rethink the architecture design of future networks and how to adjust the testing, implementation, and operation of these systems accordingly. The following gaps represent a small fraction of the measurement and technology development challenges identified by the working group related to network performance.

\subsubsection{Measurement architecture}

Working group participants noted that the research community does not agree on how to measure radio frequency usage in certain future environments. The challenge of measuring RF usage in urban, suburban, or rural settings will become more complex as systems increasingly rely on mmWave frequencies to provide additional capacity and bandwidth, since propagation characteristics in these frequencies are only beginning to be characterized and modeled. There is also a lack of understanding of how equipment will behave in colored and reactive colored noise environments. By creating controlled, multi-dimensional testbeds that extend beyond traditional metrics such as gain and direction of incoming transmissions will make it easier to know and predict when antennas will be impacted by environmental factors.

Measurement architectures will need to be designed to more precisely measure whether networks are directional, what direction information is coming from, and how well devices are measuring directionality. Measuring range and direction accurately in cluttered environments and developing standards for evaluating equipment in the face of colored noise will become increasingly important for data systems using antennas. Several working group members stated that there are many more questions than answers in this space currently.

\subsubsection{Protocol performance}

The working group argued that one of the key evaluation challenges facing industry is the critical need to develop more simple, straightforward ways to measure network protocol performance, especially given the mission critical nature of many future generation wireless applications. For example, future generation wireless application areas such as remote surgery or autonomous vehicle operation will require minimal latency. Since a single point of failure in these future services would present significant risk to the safety of both users and operators of these applications, industry needs to develop protocols that can more accurately and confidently test requirements such as latency before these applications will be adopted widely.

One participant described the lack of "simplicity" of measuring protocol performance today either in terms of implementation complexity or some more abstract measure (e.g., state diagram). For example, some transport protocols, performance measured in fraction of available bandwidth occupied (efficiency) is relevant, but this is not a metric for control and signaling protocols. In other cases, delay, measured in round-trip times, may be a concern, particularly if negotiation is required.

Several participants conceded that much of industry is simply not aware of what barometers are being used to measure protocol performance today. Apart from the Internet Engineering Task Force's (IETF) Internet Protocol (IP) performance metrics (IPPM) working group that has been developing standard metrics and protocols to measure IP performance, there are very few widespread standards or benchmarks to assess protocol performance. Protocol 
research is usually not done on a standard network, which makes it impossible to compare protocols meaningfully. The networking community would benefit by defining sets of networks and traffic models to be used as benchmarks. With this in mind industry needs to establish more commonly understood techniques to measure latency, total throughput, aggregate throughput in a multi-user environment. Although there may be accurate ways to measure protocol performance today, working group participants were not able to identify any agreed upon techniques currently used across industry stakeholders.

\subsubsection{Test and evaluation}

The working group discussed that industry needs to develop and standardize more sophisticated test equipment in the lab and in the field. However, before a measurement procedure can be standardized, researchers need to understand the requirements of the system for which the test is being designed. Manufacturers design testing procedures based on antenna parameters established in the standard. Therefore, industry needs to standardize testing methods before it is able to accurately predict the performance of this standard in real world deployments.

Today industry primarily tests devices with white noise, but "real world" noise actually has color. Some noise, such as phase noise is an undesired by-product of a transmitter which is intentionally transmitting. Other noise, such as ignition noise, represents unintentional transmission. These should be distinguished from out-of-band emissions and spurs which are noise-like and are typically deemed interference. Furthermore, researchers will need to pursue fundamental measurement and modeling science to develop a better baseline understanding of how to characterize these highly-colored noise environments.

Today's simulation models also fail to account for colored noise, and new measurement techniques need to be developed in light of these modeling limitations. Industry cannot always run models in a full range of spectrum locations in the "real world," and therefore a profile for colored spectrum is needed to make lab experiments more realistic. In mmWave frequencies environments, the sources of interference will be highly directional, colored, and potentially frequency selective. Every dimension of space-time frequency will be local. This reality represents an environment that is as far away from "white" noise as possible. As a result, industry needs to advance modeling in the area of colored interference profiles.

\subsubsection{Machine Learning}

Expanded use of network analytics will provide more granular visibility and control of how devices will communicate on future wireless networks. This capability will prove critical as network operators strive to provide a greater diversity of sophisticated, data-intensive applications and services across larger network coverage areas. The working group consistently cited that advances in machine learning, artificial intelligence (AI), and algorithm development could dramatically improve the way that future generation networks manage resources and self-optimize in relation to shifting traffic and demand patterns. While integrating data science techniques to a greater degree in network planning is anticipated to be a key driver of improved network performance, spectral efficiency, and power consumption; several technology development gaps need to be addressed before these benefits reach commercial scale. 


\subsubsection{Data mining}

Today's AI control methods require the central decision maker within the network architecture to possess the same data that exists across all other agents in the system(s) to make consistent conclusions. This results in extreme amounts of data transfer and a massive data crunch.

Efforts are underway today to reduce the amount of data being transmitted to a centralized decision making point in the network, but the working group could not identify solutions available in the marketplace today. Reducing transmission latency and the volume of data transmitted between network endpoints is a prerequisite for introducing AI concepts into the automation of network management. While many stakeholders see the "decentralization of networks" as a key enabler of future generation wireless capabilities, introducing more distributed architectures does not solve the problem of higher-transmission times. There are many distributed components in the control plane, including base station, device, mobility management, and all need to come to a consensus to run properly, which is time consuming. Separating the control and data plane helps somewhat, but there are still inconsistencies and need additional messaging for achieving consensus and properly managing the data flow.

\subsubsection{Resource management}

The ability to flexibly manage bandwidth resources by adapting to changing usage patterns is seen as a key differentiator for future wireless networks. Data scientists need to develop new algorithms that can automatically prioritizing data transmission across multiple network layers based on geographic proximity, variations in upload and download traffic, and dynamic performance requirements; future wireless networks will gain the ability to selforganize. Additional algorithm capabilities need to be developed that can automate load balancing, minimize interference, and allocate spectrum across network nodes. Analytics will help maintain service quality and meet performance demands even when networks are at capacity or under stress.

Future generation networks will also be expected to more precisely adjust the quality of audio, video, qualitative text, and statistical data in real time. Developing algorithms, tripwires, and data governance processes that can evaluate and make decisions based on a given system's bandwidth constraints with user's data quality requirements will be critical in maintaining expected quality-of-service during times of network stress, saturation, or periods of peak data demand.

In addition, industry will need to evaluate how protocols will allocate different amounts of data to multiple users with multiple devices given their varying data demands. Developing new protocols that can deal with asymmetry - varying "Uplink vs. Downlink" data demand was also cited as an important area of future research for optimizing resource allocation.

Researchers also expect machine learning algorithms to play a critical role in optimizing the efficiency of network transmission power management. Future generation wireless systems will be characterized by ubiquitous machine-to-machine (M2M) communications, and these localized internet-of-things networks will rely on passive, low-power, "always-on" connections. Industry has divided the future of M2M use cases into two primary categories. The first is often described as Massive machine-type communications (MTC), which relates 
to communication between a very large number of low-cost, low-data, and low-power devices such as utility meters. The scalability of network architecture is the primary concern in preparation for future Massive MTC deployments. The future devices that constitute Massive MTC deployments will also remain in service for long periods of time and will need to function for years or even decades with minimal downtime due to power outage, battery expiration, or maintenance issues. The second category, Mission critical MTC, relates to services that will likely need to adhere to rigorous latency and reliability requirements such as industrial process automation and driverless cars. Today's power management methods will need to be bolstered by more sophisticated machine learning algorithms and data science techniques to ensure that mission critical elements of systems supporting applications such as robotic surgery, vehicle-to-vehicle communications, and public safety communications with building or municipal utility controls do not fail due to these devices consuming too much power.

Lastly, many researchers maintain a system-level approach for reducing the impact of interference that would be greatly aided by expanded use of intelligent machine learning algorithms as discussed in the spectrum sharing section of this document.

\section{Conclusion}

The Future Generation Wireless R\&D Gaps Report represents a culmination of a year-long engagement with leaders in the telecommunications industry - spanning from private companies, research institutions, and standards bodies. NIST greatly appreciates the invaluable contributions made by working group members that helped develop this final document, and is committed to continued outreach with wireless stakeholders when updating or building on this report. The gaps identified in this report present a wide range of ideas, challenges, and opportunities for the wireless research community to consider. NIST will use these findings as a resource to assist with its own research planning activities and to provide maximum benefit to its stakeholders. 


\section{Appendix A. Research Methodology}

As outlined in the Research Design section, NIST deployed a deliberate, systematic approach to stakeholder engagement and data collection to develop this report. The steps NIST used to evaluate the gaps and challenges associated with future wireless technology are described below.

\section{Timeline}

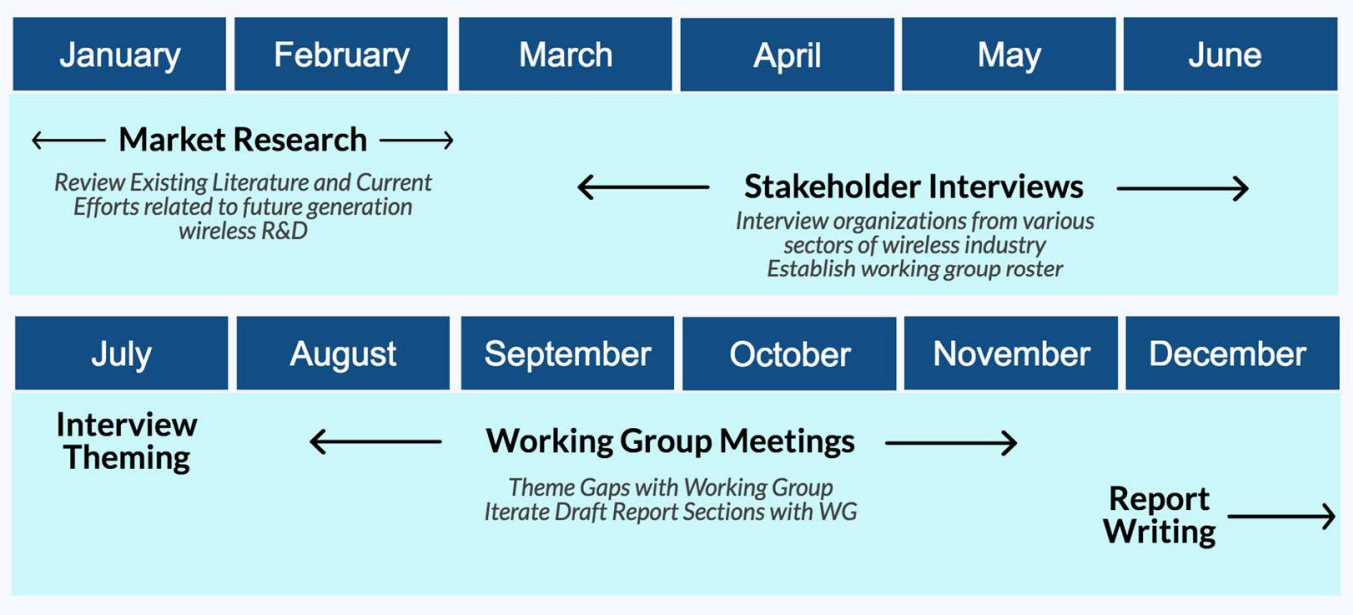

Fig. 3. 2016 Report Development Timeline

\section{A.1. Market Research and Stakeholder Interviews (January - July 2016)}

First, the team conducted an intensive review of existing research literature describing the vision for $5 \mathrm{G}$, expected performance requirements, and potential challenges or technology gaps that needed to be overcome. NIST reviewed materials such as white papers from standards bodies, corporate white papers, industry journals, and editorial content to develop an initial diagnostic survey of the key components and research priorities of the changing wireless industry.

After this preliminary step, the team conducted a series of one-on-one interviews with key stakeholders to build on the findings of the market research report. NIST interviewed a total of 27 people representing organizations across private industry, the federal government, academia and non-profit research consortia. The purpose of the interview process was to collect more specific data related to the organizations' current research activities, short and long-term R\&D challenges, and requirements for future wireless systems. NIST also polled the organizations' willingness to participate in the upcoming working group during these interviews and collected information on other stakeholder groups to target.

\section{A.2. Working Group Meetings (July - December 2016)}

NIST engaged a sufficiently diverse stakeholder audience to accurately characterize specific challenges within the rapidly evolving future generation wireless technology sector. NIST built on existing relationships, survey data, and literature review while developing the Market Research Report to identify specific measurement, technology, and management gaps within each component of the wireless industry (framework verticals). To collect the data to be included in this report, NIST leveraged both conference calls and an interactive wiki platform 
that prioritized the efficiency of time and resources of participants and promoted transparency and traceability throughout the report development process:

- Efficiency of time and resources - Deploying a set of digital tools on a wiki platform enabled stakeholders to provide direct, first-person input and document their expertise at their own convenience. Pairing digital forms, meeting minutes, and discussion boards with conference calls and stakeholder interviews allowed the working group to spend valuable meeting time validating and contextualizing input rather than collecting data on the fly. also minimized the risk of transcription error while collecting data and allowed participants to view and comment on their peers' form submissions.

- Traceability \& Transparency - NIST captured stakeholder input at each stage of the engagement process and published this information to the group between meetings. This iterative provision of data helped document the logic leading up to these findings, minimized the risk of transcription error while collecting data, and allowed participants to view and comment on their peers' form submissions.

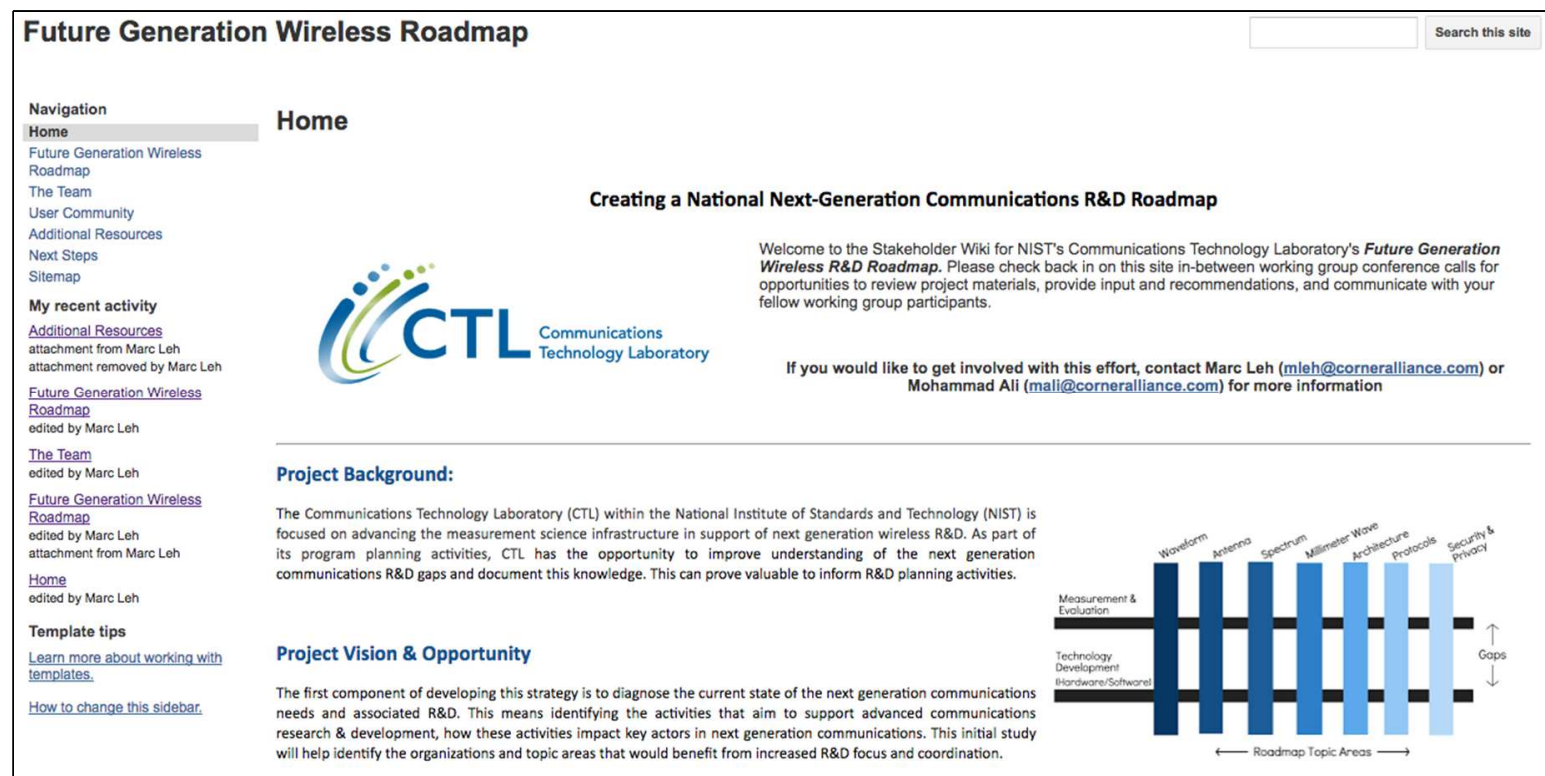

Fig. 4. Working Group Wiki Platform

The working group met biweekly to discuss each vertical of the framework. In addition to identifying measurement, technology development, and management gaps associated with the vertical next to be discussed, working group participants provided feedback and refinements to documents summarizing past calls throughout the meeting schedule. This iterative workflow allowed the NIST team to identify gap themes that spanned across specific verticals. Given how interrelated many of the topics are to one another, these gap themes yielded a more readable final product, and added a second layer of analysis to original data collected. The R\&D Gaps Framework that helped focus working group input on 
the wiki and corresponding conference call meetings is illustrated in the Figure 5.

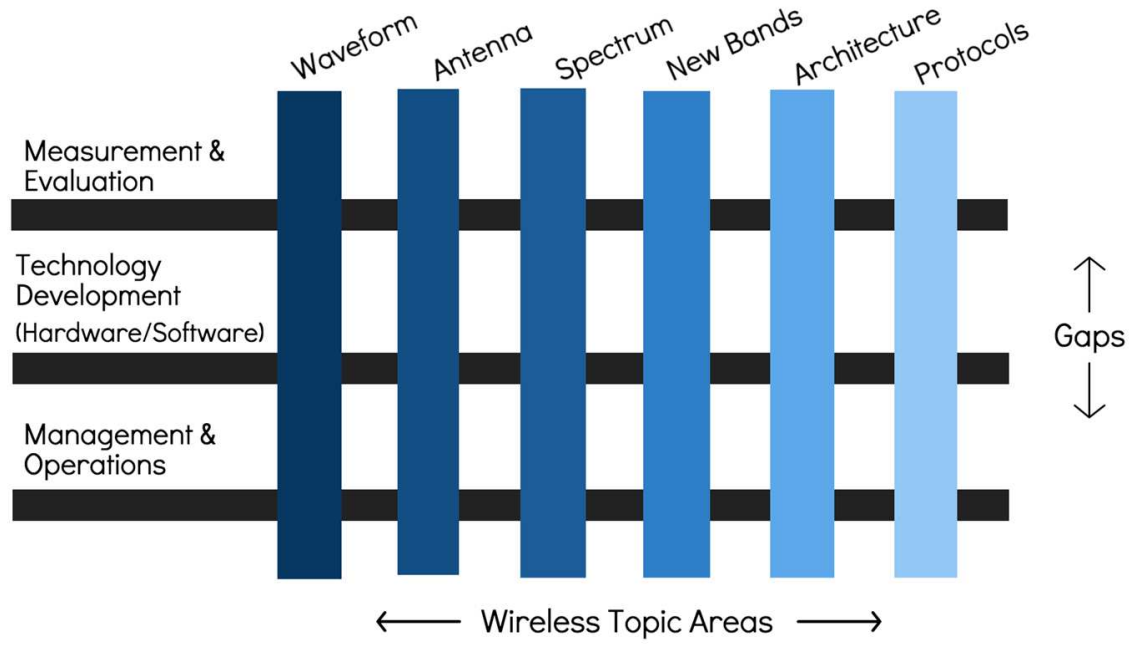

Fig. 5. R\&D Gaps Analysis Framework

\section{A.3. Working Group Roster}

The volunteer working group members were instrumental in developing the findings and conclusions described in this report. NIST greatly appreciates the time, commitment, and expertise each was able to provide in service of this effort. A complete roster of working group participants can be found in the Table 1.

Table 1. Working group Roster

\begin{tabular}{|l|l|l|}
\hline Name & Organization & Discipline \\
\hline 1. John Chapin & Carnegie Mellon & Academia \\
\hline 2. Henning Schulzrinne & Columbia University & Academia \\
\hline 3. Thyagarajan Nandagopal & FCC & Academia \\
\hline 4. Dipankar Raychaudhuri & Rutgers WinLab & Academia \\
\hline 5. K. K. Ramakrishnan & University of California-Riverside & Academia \\
\hline 6. Victor Berrios & ZigBee Alliance & Academia \\
\hline 7. Joe Evans & DARPA & Government \\
\hline 8. Paul Tilghman & DARPA & Government \\
\hline 9. Brian Fox & U.S. Department of Defense & Government \\
\hline 10. Frederick Moorefield & U.S. Department of Defense & Government \\
\hline 11. Robert Schneider & U.S. Department of Defense & Government \\
\hline 12. Michael Ha & FCC & Government \\
\hline
\end{tabular}




\begin{tabular}{|c|c|c|}
\hline Name & Organization & Discipline \\
\hline 13. Anoop Gupta & Amazon & Industry \\
\hline 14. Craig Partridge & BBN & Industry \\
\hline 15. K Claffy & Center for Applied Internet Data Analysis & Industry \\
\hline 16. Aleksandra Boskovic & Corning & Industry \\
\hline 17. Michael Yadlowsky & Corning & Industry \\
\hline 18. Waguih Ishak & Corning & Industry \\
\hline 19. Masoud Olfat & Federated Wireless & Industry \\
\hline 20. Andrew Clegg & Google & Industry \\
\hline 21. Preston Marshall & Google & Industry \\
\hline 22. Clara Li & Intel & Industry \\
\hline 23. Geng Wu & Intel & Industry \\
\hline 24. Moray Rumney & Keysight & Industry \\
\hline 25. Paul Kolodzy & Kolodzy Consulting & Industry \\
\hline 26. Matt Ettus & National Instruments & Industry \\
\hline 27. Amitava Ghosh & Nokia & Industry \\
\hline 28. Tim Thomas & Nokia & Industry \\
\hline 29. Tod Sizer & Nokia Bell Labs & Industry \\
\hline 30. Farookh Khan & PHAZR & Industry \\
\hline 31. Oren Eliezer & PHAZR & Industry \\
\hline 32. Rakesh Taori & PHAZR & Industry \\
\hline 33. Sid Balasubramanian & PHAZR & Industry \\
\hline 34. Upkar Dhaliwal & Phluido & Industry \\
\hline 35. Jim Lansford & Qualcomm & Industry \\
\hline 36. Boon Loong Ng & Samsung & Industry \\
\hline 37. Charlie Zhang & Samsung & Industry \\
\hline 38. Joe Lipowski & Starry & Industry \\
\hline
\end{tabular}




\begin{tabular}{|l|l|l|}
\hline Name & Organization & Discipline \\
\hline 39. Gaurav Bansal & Toyota InfoTechnology Center & Industry \\
\hline 40. Roger Melen & Toyota InfoTechnology Center & Industry \\
\hline 41. Chris Murphy & ViaSat & Industry \\
\hline 42. Erwin Hudson & ViaSat & Industry \\
\hline
\end{tabular}

\section{Appendix B. Appendix B: Full Gaps List}

\section{B.1. Waveform (August 24, 2016)}

The Future Generation Wireless R\&D working group has defined the "Waveform" vertical as methods of representing information as "groups of radiating electromagnetic signals". This vertical can also be more broadly referred to as "Over the Air" (OTA).

Note that the term "waveform" has different meanings to different communities; waveform is only one aspect of putting a signal "Over the Air." In addition, emerging technologies such as MIMO combine waveform with spatial processing. Waveform, modulation, signal processing, and decoding are all in scope for this effort, which is more accurately captured by the term OTA. It is understood that this section and related gaps will have significant overlap and/or impact on the "Antennas" vertical.

Gaps within this topic area include the lack of commercial development in bands from $70 \mathrm{GHz}$ to $300 \mathrm{GHz}$ between measurement/evaluation and technology development. The gaps related to waveforms suitable for the $100 \mathrm{GHz}$ to $300 \mathrm{GHz}$ frequency regimes are even wider.

The measurement, technology, and management gaps detailed below represent a summary of working group discussions in August 2016.

1. Improved methods and tools to design waveforms and assess waveform performance

As new waveforms are developed, advanced tools, models, testbeds, and fundamental mathematical techniques to analyze waveforms will need to be developed as well. This will allow the community to understand how well each waveform facilitates efficient and effective use of the spectrum, in addition to deriving any fundamental limits that exist for new waveforms.

New waveforms will need to be analyzed in highly-colored noise environments, not white noise environments. Therefore, realistic noise models that take into account man-made noise and accurately characterized interference are needed.

In addition, more realistic propagation models are needed in order to better benchmark new waveform and system designs. 
In addition, models that capture the spatial statistics and diversity of the environment (often referred to as clutter) are needed in order to accurately characterize the waveform performance across a variety of places and environments.

Existing standards are not sufficient to accurately specify waveforms. Although 3GPP has developed definitions and specifications in this space, they are not robust or inclusive enough to inform system design or modeling tools.

Future generation wireless communications systems will interact with each other in very complex ways; there is a need to better understand how certain OTA design choices impact coexisting systems (interference, performance, etc.) in shared spectrum scenarios. Traditional metrology is limited in this regard; technology is moving faster than interference measurement methods. One example of this gap is the ongoing discussion and disagreement around whether LTE in unlicensed spectrum (LTE-U) is harmful to WiFi or not.

The ability to map the optimal choice waveform to the frequency band of operation needs to be developed.

Different waveforms are preferred in different frequencies; for frequencies over $40 \mathrm{GHZ}$ or $100 \mathrm{GHz}$, it is unknown which kinds of waveforms or modulation choices will be optimal. Although standards development organizations are focused on this space, they are primarily focused on frequencies below $100 \mathrm{GHz}$.

In addition, there is a need to study the choice of non-orthogonal modulation schemes (NOMA) with respect to waveform design at various frequency regimes. It is unknown how different modulation methodologies (interleave-division multiple-access (IDMA), etc.) will be applied to high frequencies

\section{Waveform Technology Development Gaps}

Novel waveforms are needed in order to facilitate spectrum sharing.

From a waveform development standpoint, there is a need to accurately "program" spectrum for transmission. Evaluating waveforms in modulation software does not accurately address the real propagation effects, which limits the design and the waveform technology development. RedHawk works reasonably well as a way to program the spectrum but is reception only.

Ultimately, the key challenge is to achieve a "tight burst" - where the duration, spatial spread, frequency spread, and power level of transmissions are minimized.

In a resource-limited environment, it is imperative that systems are designed to be very tight.

Advanced filters will need to be designed that ensure that the power is only utilized during transmission. Filters can ensure that when there are no transmissions, the power level goes to zero.

In general, there is less power and resources available to accomplish traditional communications tasks (transmit/receive, etc.), meaning that systems will need to be more efficient than ever before. This will involve the development of optimized modulation methods and spatial processing and coding methods that are dynamically integrated into the 
current channel as well as nearby networks. Appropriate metrics used in characterizing efficiency include (duration $\times$ space $\times$ frequency $\times$ power) divided by the tasks accomplished.

Waveforms that lower the Peak-to-Average Power Ratio (PAPR) to be at least as good as Quadrature Phase Shift Keying (QPSK), especially in frequencies about $100 \mathrm{GHz}$, terahertz, and optical, need to be determined, designed or both.

Data converters are inevitable in any system, and their performance fundamentally sets the achievable performance and bandwidths. Novel waveforms will eventually lead to waveform-system co-design to enable innovative communication systems across application areas. Systems will need to be designed with new waveforms in mind and vice versa.

\section{B.2. Antenna (October 7, 2016)}

The Future Generation Wireless R\&D working group has defined the Antenna vertical as "methods for transducing information between radiating electromagnetic signals and wired representations suitable for generation or detection." For example, the working group cited the need to develop massive numbers of antenna elements for Terahertz band communications between $(300 \mathrm{GHz}$ and $3000 \mathrm{GHz})$ as an important measurement and evaluation gap facing the industry in the years ahead.

The measurement, technology, and management gaps detailed below represent a summary of working group discussions in October 2016.

3. Need improved evaluation of beam acquisition and tracking at both ends of the link in a dynamic 3D environment that includes realistic interference, multi-band phantoms, blocking, shadow fading, and in-channel impairments.

The working group cited that it might be challenging to measure or evaluate the effectiveness of beam-steered antennas as the dynamics of that capability is beyond today's measurement techniques for conventional antennas with fixed/static radiation patterns. The working group feels that the rate at which the radiation changes in non-static antennas with variable radiation patterns will be an important research topic. Radiation can vary according to beam width or steering, and today's laboratories do not have effective measurement methods.

4. Need to develop more effective forms of dynamic and intelligent beam-steering, as this will offer a relief for high propagation losses experienced in millimeter-wave bands, while also mitigating interference and allowing better spectral reuse, resulting in highernetwork capacities.

The working group also posited that next generation antennas will have variable radiation parameters, such as beam width, radiation patterns, and directionality. One has to be able to use these variations (in beam width and steering) in order to maximize capacity and reduce interference. In spectrum sharing environments, transmitter nulls will be relied upon heavily to reduce interference at receivers in fixed locations. However, developing an optimal nulling strategy today remains hard and limited by the tools available to characterize and assess interference.

5. Complex measurement/validation setups are needed for future antennas 
As antenna systems become more complex over time, offering targeted rejection of interferers (null-steering), they will require even more complex measurement and validation setups. One specific challenge is that characterizing a null (rejection) means characterizing a loss, and that there is potential for multipath in these measurements.

Another challenge cited by the working group is that conditions in an anechoic chamber do not reflect what they would be in the field. Sterile, anechoic measurements do not represent antenna behavior in a dense urban environment. Antennas in the field, especially ones used in handheld devices are highly affected by the environment.

Test setups are needed to accurately and reliably emulate what happens in the field and for different mounting scenarios. The FCC tests various devices by emulating the conditions that the product will be placed in when sold. These conditions are not always entirely accurate for real world deployment. Additional environmental interference testing is often needed. Thus industry needs new ways to simulate interference for different deployment environments.

Given this future environment, researchers need to consider developing standards for the antenna's context of use and when to test antennas in a deployment environment rather than in a lab.

6. Need for standardized testing and specification of millimeter-wave antennas, especially phased arrays.

The working group discussed that industry needs to develop and standardize more sophisticated test equipment in the lab and in the field. However, before a measurement procedure can be standardized, researchers need to understand the requirements of the system for which the test is being designed. Manufacturers design testing procedures based on antenna parameters established in the standard. Therefore, industry needs to standardize testing methods before it is able to accurately predict the performance of this standard in real world deployments.

The working group did point out that while standards do exist for antenna performance today, they do not exist for the architecture of the antenna. Usually standards accommodate several architecture options, but they do not specify what industry needs to use. 3GPP is addressing this gap for mmWave.

7. Need for standardized interfaces and connections for automated tests of mm-wave antennas.

The working group emphasized the need for standardized interfaces and connections for tests of mmWave antennas, and that a strong "gold standard" would benefit industry well into the future. The gap here is the lack of the standard testing and a need for methods to specify and test antennas.

8. Need hardware and software developments allowing for low-cost and power-efficient phased-arrays to realize a capacity maximized network.

Need to develop active antenna hardware and algorithms to control them, particularly in the presence of UE head and hand phantoms. 
Over time, the working group expects performance metrics to be tightened (rate of adaptation, beam-widths, gain-to-rejection ratios, etc.) as capacity demands on future generation networks increase. "Capacity Maximized" was defined by the working group as the ability to build antennas with tunable frequency nulls that result in more capacity. Some working group members believe that the tunable nulls technique for maximizing network capacity may be impractical today, but not impossible into the future. If researchers need to nullify frequencies, some argued, they could do it in analog rather than in a digital system setup. They would also need to build in a wide range into the transceiver electronics to effectively tune nulls.

Others pointed out that nullifying signals close in frequency is a practice currently used by the military to prevent jamming. These examples use radically different, non-traditional antenna designs for transducing electromagnetic radiation. They are trying to achieve the ability to reject incoming signals that are close in frequency and that are beyond a certain dynamic range of an a-to-b converter. For example, a "notch filter" is built into the antenna to support desired behavior, but the implementation of this is entirely different. Signal processing techniques and spatial can also help discriminate the null. The working group also noted that is unclear how much the commercial community is looking at this, and how practical this technique would be in industrial or commercial deployments.

9. Need improved development of wideband (multi-octave) directional antennas that are compact, lightweight and rugged.

The gap is likely to increase over time as systems are developed that integrate low, mid, and high band (mmWave) frequency capabilities.

10. Lack of electrically tunable and steerable antennas

New technology designs that have rapidly-tunable notches in frequency and spatial response in order to operate better in congested spectrum do not exist in commercial deployments today.

11. Need for improved antennas with higher gain over broader frequency ranges.

This has been a common technology gap over the past 20 years.

12. Need for mobile device that can support simultaneous transmission and reception over a very wide frequency band (e.g., $500 \mathrm{MHz}$ to $100 \mathrm{GHz}$ ).

There is a need for managing antennas (or a group of antennas) that can support such wideband operation (e.g., maintain consistent array performance despite the vastly different wavelengths) and enable small form factor at mobile device (e.g., integration of different antennas for different bands in a small form factor).

13. As higher frequencies are utilized for cellular, the number of antenna elements increases, but the number of RF chains is limited due to power consumption.

The working group argued that there is a need to study the optimal massive antenna array architecture with limited RF chains and power consumption. There is also a need to research how to efficiently integrate the antennas with Radio Frequency Integrated Circuits (RFICs). 
This technology gap is receiving a lot of attention in academia and industry today. For example, Massive MIMO technologies are the subject of countless publications and demonstrations today. Current challenges related to this gap arise in multiple domains such as circuitry, signal processing, power dissipation, and impractically large physical size (distance between antennas needs to be relatively large in relation to wavelength).

The solutions in this space may be very promising, but it is not well known when they will be deployed or how practical they will be. Some solutions include full duplex antenna architecture or the significant rejection in analog circulators. Columbia University is developing applicable technology today. When commercializing these solutions, low-cost design will be essential to mass adoption and achieving spectral reuse benefits.

14. Need improved validation to ensure that field performance is approaching optimal performance - i.e., how to troubleshoot issues.

The working group identified the need to monitor antenna performance in a variety of usage scenarios; we need to be able to recognize and monitor degradation. Specifically, the challenge in this space is related to the directionality of the coupler. Chips and signal processing can help mitigate this challenge in handsets to some extent.

\section{B.3. Spectrum (September 9, 2016)}

The Future Generation Wireless R\&D working group has defined the Spectrum vertical as "Methods for coexistence of independent systems that use radiating electromagnetic signals; understanding factors in the electromagnetic environment that affect the performance of such systems and their coexistence." For example, the working group cited the lack of commonly accepted coexistence performance metrics to evaluate effectiveness of spectrum sharing protocols as an important measurement and evaluation gap facing the industry in the years ahead.

The measurement, technology, and management gaps detailed below represent a summary of working group discussions in September 2016.

\section{Need for improved measurement environments \& testbeds}

There is a need to conduct spectrum measurement and evaluation in more realistic "real world" environments. Today industry primarily tests devices with white noise. But noise actually has color and we are moving to a world in which the noise is smart, that is the device that is generating noise can realize that it is doing so. Thus, there is a need to establish what types of noise and intelligence of noise is sufficiently realistic for future generation spectrum testing. A distinction can be drawn between white noise, or channel impairments, and true sources of interference, which include non-communication signals (i.e., motor, ignition noise). Communication systems are stationary and have predictability in their characteristics. On the other hand, the non-communications interference sources are less predictable. The measurement stream should differentiate between intentional radiators, man-made noise, natural noise, and intermodulation spurs.

In mmWave frequencies environments the sources of "noise" and interference will be highly directional and colored (potentially frequency selective). Every dimension of space, time frequency will be local. This is as far away from "white" as we can get. Need to advance 
modeling in this area and develop colored interference profiles. There are no spatial test cases available today to deal with this type of interference.

In addition, communications in the millimeter-wave bands will be highly local. Therefore, real world measurement will be done by the devices themselves and their neighboring devices -- so there is a modest data flows from a large number of uncalibrated devices that need to be considered.

In summary, we lack accurate models that can account for colored noise. How do we measure against this reality? We need a profile for colored spectrum to make lab experiments more realistic.

The working group highlighted several measurement and evaluation gaps that are specific to future generation wireless system deployments in the field. These include the need to achieve denser coverage in time space and frequency in congested areas at affordable price. These environments include high-density urban areas, outdoor-to-indoor system architectures, and high-density indoor spaces such as offices, stadiums, and shopping malls. The group also observed the need to measure anomalous propagation conditions (e.g., ducting) sufficiently to provide statistically valid characterization their likelihood in specific areas or channels.

There is currently a shortfall of testbeds that can be used to evaluate spectrum sharing, efficiency, flexibility, and agility (especially in heterogeneous electromagnetic environment consisting of military, government and commercial wireless systems.)

Need to develop large-scale testbeds that reflect real-life scenarios to evaluate co-existence performance.

The NSF's PAWR Projects may have begun answering this need. The wireless cities initiative (PAWR) is proposing to wire up four cities, and that carriers participating in this environment need to capture realistic workloads. This means that these testbeds will not simply drive around with a breadboard. These next generation testbeds would need to enable users to use the technology in real world scenarios such as dense urban environments vs. suburban or rural areas will have spectrum behave in different ways.

FCC's program license makes this challenge of simulating real world scenarios and usage easier. Although the FCC program does not qualify as "large-scale" in terms of featuring a lot of real world users, its use in a lab environment is helpful in solving the cooperative challenge in working environments.

The DARPA spectrum collaboration challenge is developing another lab-based testbed that could be similarly useful in simulating real world scenarios.

16. Need to develop improved metrics to evaluate spectrum sharing

The working group emphasized the need to establish new metrics to evaluate the efficiency of spectrum sharing. Evaluation metrics should drive the evolution or revolution towards better spectrum sharing technologies.

In future communications environments, signal interference will increasingly affect how we measure things. If there is a group of co-channel users, we will have more direction on how 
to share spectrum. Metrics need to account for what is better for the full group, by incorporating principles related to gain theory for example, in terms of throughput opportunity cost for individual vs. group users. The group also emphasized the need to look specifically at developing metrics for group optimization concepts.

In addition, there needs to be a metrics for multiple sharing dimensions. Since sharing may not be possible with all protocols, there needs to be metrics that capture who is asking for it and what tasks they are performing and what they care about.

For example, regulators are technology agnostic, and need metrics that characterize the entire system. On the other hand, standard bodies want to know what is the best metric for spectrum sharing efficiency for a certain technology (e.g., WiFi Alliance is looking to understand the metrics related to backhaul and how WiFi is affected by LTE-U/License Assisted Access (LAA)).

Another important point is to develop commonly accepted co-existence performance metrics to evaluate effectiveness of spectrum sharing protocols, especially for systems with equal priority of spectrum access.

Finally, the group indicated that the research community needs to fundamentally rethink the notion of what it means to "use" spectrum. One specific area of need is improved methods to define whether a frequency is "used" at a specified time and place. Other gaps within spectrum sharing and monitoring include developing improved methods to define whether or not a system was interfered with; the need to develop new propagation models that are valid out to high path loss levels; and the need to develop models of aggregate interference behavior from large numbers of spectrum users.

Since there is no single number that can be used to characterize interference, there needs to be a set of metrics that are developed so that new receivers can be added into the time/location/frequency without addition interference. Currently this is hard to achieve with MIMO and advanced signal processing.

\section{Gaps in spectrum sharing scenarios studied}

The group identified that signal co-existence and interference between $5 \mathrm{G}$ and fixed systems at various mmWave spectrum bands would be a significant gap going forward. One such example of this challenge is the coexistence of $5 \mathrm{G}$ at $28 \mathrm{GHz}$ with satellite systems. Researchers need to study interference in this scenario given the expanded use of satellite networks going forward.

Also given that future generation wireless will feature a lot of point-to-point backhaul at higher frequencies, industry needs a better understanding of the interference challenges at frequencies above $60 \mathrm{GHz}$ or $100 \mathrm{GHz}$. This is a bigger challenge in Europe than it is in Asia. Research organizations in the United States have done studies on what types of isolation is needed, but at the mmWave band researchers need additional study on interference between existing links.

18. Gaps in design, validation and testing methods 
Relying on large antenna arrays at both ends of the link to operate $5 \mathrm{G}$ in the lower mmWave bands (28 GHz and up) will disrupt everything industry knows about design and validation. Future testing will have to be radiated, with the most important tests being those for signal acquisition and tracking in a dynamic 3D environment including body shadowing and blocking effects - none of which are available today.

The group emphasized that future systems will only need the large antenna array at one end of the link, and that systems cannot assume that the power level closer to the transmitter will always be higher than it is farther away. Need to measure the transmitted energy of a system that uses MIMO technology and spatial processing in complex propagation environments to focus its energy at points away from the transmitter. The power level of a transmitted signal is no longer a decreasing function of distance.

Current spatial testing technology does not scale to mmWave. Signals trying to create are narrower anyway, so the use of anechoic chamber may provide some answers. This challenge is just starting to be discussed at $3 \mathrm{GPP}$.

\section{Technology development gaps}

Industry lacks a standard hardware/software platform for collaboration and common programming languages and libraries for programming waveforms. Working group participants complained that they and their peers cannot display or share solutions on a standard platform in a way that industry colleagues can use today, especially in lab settings. Standardized collaboration platforms would serve as a valuable tech transfer vehicle. Although some in the working group saw collaboration platforms as an aspirational - albeit valuable - gap to improve technology transfer.

The working group emphasized that industry cannot effectively manage interference between various systems at different spectrum bands (especially at higher bands) due to highly directional nature of the antenna arrays at these frequencies. To help address this gap, the research community need to develop receiver circuits and filters that can reconfigure themselves to remove intermodulation out of the passband

There is a need to develop cheap, small, high quality filters that are frequency tunable.

With this technology, we could fit more users into the spectrum without adding unnecessary complexity elsewhere in the system. We have great signal processing but it is difficult to do software-defined filters.

There is also a need to develop cost-effective steerable antennas for mobile devices and the algorithms to control them at both ends of the link to overcome dynamic 3D fading/blocking. Today we can only create $2 \mathrm{D}$ spatial fields. This works at low frequency, but at mmWave the ability to control the wave fields is a function of angular relationship of antennas.

In order to get the link budgets working well, steering is required at both ends of the link. Mobile will have a more difficult time steering than the base station. Needs to also account for hand and body shadowing. A greater deal of agility in antenna design is needed to match that of a base station. 
Also, there needs to be more sophisticated transmission power management methods and other technologies to improve spectrum sharing.

Many researchers maintain a system level approach for reducing the impact of interference including collaborative and non-collaborative approaches. Non-collaborative approaches are more difficult to achieve since systems are not able to communicate with one another. Also, there may be a coexistence and a conflict of interest between the two systems when they are collaborating.

When collaboration channels are present, industry needs to determine the information that should be shared among networks for collaboration purpose to facilitate efficient coexistence. The working group envisions that the Information to be shared may within these collaboration channels may include medium access control (MAC) and physical layer (PHY) information and the rate/condition of information exchange. Some participants indicated that it will be easy to overwhelm the coexistence channel in future generation systems. If you have a collaboration channel, operators need to be selective in what is featured on this channel. Machine learning focused on intelligence spectrum management struggles with overloading the channel. There are studies related to sharing test models or noise models that highlight the power necessary to share information.

Currently, the DARPA SC2 program is working to develop a protocol for sharing this information. Another example is the discussion in IEEE 802.11ax looking at local vs. global optimization. They sometimes have different goals. Need to look at what we are trying to optimizing (local vs. global).

There is a need to develop an integrated spectrum situational awareness, command, and control architecture. The architecture should enable controllable, adaptive, and flexible operations of hardened Spectrum Dependent Systems.

20. Spectrum management \& operations gaps

Participants recognized the need to develop a consensus or shared vision of who is managing the spectrum, is it centralized or distributed control, i.e., cell towers, WiFi hubs, or devices working collaboratively without central control?

There is a need to design appropriate spectrum access systems for both outdoor and indoor operation covering both licensed and unlicensed bands up to $100 \mathrm{GHz}$.

Deployment density needs to be overcome to provide sufficient probability of service in highly blocked environments. Improved methods of providing reliable service on top of shared spectrum are lacking.

Operations, administration and management (OA\&M) systems that are capable of: automatically detecting, characterizing and reporting system performance impacts due to RF energy incoming from independent spectrum users are needed.

Finally, there is a need to develop an electromagnetic spectrum dynamic planning, directing, and control capability to conduct real-time spectrum operations in heterogeneous electromagnetic environments. 


\section{B.4. New Bands (September 22, 2016)}

The Future Generation Wireless R\&D working group has defined the New Bands vertical as "Methods for exploiting previously unused or lightly used portions of the electromagnetic spectrum." For example, the working group cited the accurate characterization of millimeterwave signal propagation and properties, and specifically the need for improved measurement and modeling techniques, as an important measurement and evaluation gap facing the industry in the years ahead.

The measurement, technology, and management gaps detailed below represent a summary of working group discussions in September 2016.

21. Need for novel techniques to perform spectrum monitoring with increased antenna directionality

The working group cited that the proliferation of highly directional antennas means the idea of having a measuring device that can just listen to all the emitters "around" it no longer works. Measuring devices will only hear the directional antennas that choose to transmit to the measuring devices' locations. According to insight gathered from an NSF meeting from earlier this year, spectrum monitoring is executed by national spectrum measuring centers funded by the FCC. Highly sophisticated and calibrated systems are used to report on everything that the system hears. In a directional environment, this model will not work. Existing systems cannot capture a well-rounded, coherent characterization of the spectrum by only monitoring it in one place.

Specifically, the working group indicated that there is a need to gain a better understanding of what spectrum is being used or underutilized by developing new approaches to spectrum monitoring such as developing devices that could self-report what they are experiencing (including standards for self-reporting data and calibration for devices doing the selfreporting), and discovering new methods for verifying which bands these devices are using when self-reporting their experiences. These new approaches are likely to significantly change the market strategies of key telecommunications industry actors.

Spectrum occupancy measurements are typically used to determine whether new transmitters can be added without causing additional interference. In a directional world, this becomes significantly more challenging since the interference need to be assessed with respect to each receiver.

Instead of measuring the environment in advance, we want to deploy the system and turn up the operation / power and then have incumbent systems report on if and when they see it.

Location databases are one solution to addressing these gaps where interference predictions are computed in advance and relying on accurate propagation models, which may not be always available. These techniques may not be feasible for free space optical, especially in the higher-frequency bands.

22. Need to extend the range achieved with millimeter-wave bands

Working group participants agreed on the fact that millimeter-wave (mmWave) bands will enable next generation network capabilities, but that mmWave transmissions will likely only be heard by devices a short distance away. The working group expects that future devices 
may in fact be able to hear mmWave transmissions from longer distances, but the science and solutions to do so have not yet been developed. We may be able to understand mmWave transmissions better than we expect at longer distances, but additional resources need to be dedicated to this challenge of short-range mmWave bands being used in large-scale commercial networks.

23. Need for improved measurement and modeling techniques in new bands to understand the unique signal propagation characteristics across a wide range of frequency bands.

Although measurements are currently being made over all commercially-interesting bands, most sets of measurements are for a single band. The working group indicated that comparing bands at different frequencies presents many unknowns, including different equipment, procedures, and teams. Ideally, a single team with a single methodology would study multiple bands simultaneously. However, in reality, today's measurement campaigns and models track specific scenarios and frequency bands; as a result, there is a lack of comprehensive data across large sweeps of frequency bands.

The working group believes that industry is achieving better characterization of bands by a device that is designed for that band, but that these characterizations will need to be integrated with other bands to make it commercially viable. Future network environments will require multiple measurement devices that are optimized for different purposes (i.e., mobile device vs. a grounded, calibrated device) and that industry needs to develop an architecture that can handle these competing considerations. This architecture will also need to integrate measurements from different devices. At this point the working group argues that the area in most need of R\&D attention is furthering the underlying science required to fully understand the various pieces of this future architecture and developing it in a holistic manner.

Specific architecture challenges cited by the working group include:

- Metadata standards: Industry needs to more accurately capture how measurements are taken. For example, where was the antenna at the time of measurement? Better metadata standards need to be developed, which is a challenge intellectually and commercially, and requires understanding the key characteristics of various measurement systems.

- System architecture - How will industry authenticate users, networks, and devices within this future architecture? How will researchers collect measurements with an agreed upon level of trust? Where are queries made?

The working group pointed out that the astronomy community is experiencing a similar metadata challenge in terms of sharing and integrating their measurement data. Working group participants argued that the telecommunications industry could learn from this community and apply tactics to future network challenges.

24. Need for measurement campaigns and methodology that can produce commonly accepted channel models for frequency bands above $100 \mathrm{GHz}$. 
The working group indicated that 3GPP and several other industry and academic groups have studied and published channel models for up to $100 \mathrm{GHz}$ band (e.g., 3GPP TR 38.900). However, there is a lack of understanding of the propagation characteristics of frequencies above that. Participants specifically called out the need to dedicate more attention to frequencies above $60 \mathrm{GHz}$. Other challenges presented at higher frequencies include a lack of measurements and measurement data sets available from live systems or for mobile environments.

Anyone who wants to predict the network environment needs to predict how new transmissions may engage with these systems. Therefore, there is a need for measurement characterization of widely deployed multi-beam antenna arrays. Without this it will be difficult to achieve accurate modeling.

25. Need to study waveform, multiple access frameworks and other detailed system design aspects and system operations for new bands.

The working group indicated that 10 years ago industry did not have a good understanding of how to build omnidirectional networks. Directional routing today does have working algorithms, but it is not clear if these algorithms meet all requirements. These algorithms were demonstrated by DARPA about 10 years ago, and have since undergone improvements.

Directional multiple access control mechanisms may meet requirements.

In a software defined radio environment, the network layer will control the physical connection on a packet basis. This network design is directly opposite to original network design approaches developed in the 1960s and 1970s. In this new design model, the network layer can look at traffic patterns and then establish physical topology based on this data. This would present additional challenges.

Although network topology may not be well understood for directional antennas today, the working group argued that traffic-driven network topologies have been well understood from the perspective of low-energy use of networks. There is an ability to track where everyone is, and there's a process to establish links based on where packets need to go.

26. Need for new methods to extrapolate models from measurements, especially in free space optical networks.

The working group expressed concerned that consumer applications in the free space optical network space will be published before receiver filters enter the industry. There is only a narrow band where optical fiber systems can be used; without better receive filters there will be more interference. This could present a major problem and could prevent the use of cheap transmitters.

27. Lack of seamless operation of radios across multi-bands including millimeter-wave.

One of the earliest challenges that needs to be addressed for new frequency bands it to identify who is in charge of operating radios across multiple bands. Depending on a user or device's location ( $\mathrm{x}, \mathrm{y}, \mathrm{z}$ axis), the entity in charge of determining what band a user or device can use at what power and in what direction could change. The working group argues that 
industry needs to better understand what piece of equipment, organization or both determines this.

The working group also foresees the need to envision what the control channels for this look like. (i.e., in a specific area, organization/collaborative group $\mathrm{X}$ gets to decide). There is a lack of consensus in industry today. For example, when devices enter into a new environment, how can they describe their capabilities? What do the devices need to say, how much can they say, and how can we minimize what they say (to accommodate for millions of devices in a single location)? The working group also questioned whether it is possible to have multiple management domains, and what do devices do when these management domains overlap?

28. Need to determine new policies and procedures for sharing new frequency bands.

The working group raised the challenge of resolving conflicts in sharing licensed and unlicensed spectrum bands. When two groups (licensed or unlicensed) contend for the same spectrum, there is typically a primary and secondary user; future scenarios may not have primary and secondary users. This challenge is straightforward to resolve in licensed bands; existing policies and sharing techniques could be applied to new bands with relative ease. For example, primary vs. secondary use of whitespace, where primary has priority.

With new unlicensed bands, this challenge is more difficult. There is a mechanism to resolve licensed users that are in conflict - if the licensed users are licensed sequentially it could help resolve these conflicts. Prioritization can change over time. However unlicensed users do not have these mechanisms to resolve conflict. Infrastructure for new mechanisms needs to be developed to resolve resulting conflict.

Traditional spectrum management includes work on coverage optimization, but in environments where propagation is greatly affected as a function of small changes in device location it gets much harder. The working group identified the potential for a new management challenge associated with dividing continuous coverage in this new environment.

\section{B.5. Architecture (October 21st, 2016)}

The Future Generation Wireless R\&D working group has defined the Architecture vertical as "methods for designing systems that effectively exploit waveform, antenna, spectrum and protocol technologies to provide communications services." For example, the working group cited the need for a better control plane as an important architecture technology development gap facing the industry in the years ahead.

The measurement, technology, and management gaps detailed below represent a summary of working group discussions in October 2016.

\section{Lack of an operational measurement architecture}

The working group cited the lack of an operational measurement architecture as a key measurement and evaluation gap. For example, if researchers want to monitor radio frequency usage in the Washington DC metro area 15 years from now (in a world of millimeter-wave), there is not an agreed upon answer for doing so. The working group noted that the communications industry does not know how it will understand the RF of mmWave 
bands. These gaps will make it difficult to build a centralized architecture in urban environments and understand RF. Various solutions have been suggested but industry has not made progress toward these solutions and there is no agreement on the research problems to address in this space.

30. Lack of standards to evaluate equipment in the face of colored noise, much less reactive colored noise.

The working group specifically argued that standards that address how ambient RF environments evolve in response to tested devices need to be developed. Several participants pointed out that NIST could make an impact very quickly in this gap by developing a 2 to 3 year plan to issue colored noise and reactivity standards. The industry also needs more basic testing to provide additional information on how devices will behave in this environment.

Current research efforts in this space include safety applications and antenna technologies that use equipment to improve range and direction and go beyond basic communications applications. These efforts have raised concern over the abilities of equipment to tell which directions information comes from. This concern relates to both the need to know whether the network is directional and how well a device is measuring directions. The working group argued that the direction of information is important to know how to characterize when antennas are impacted by environmental factors, for example, and whether the device is measuring radio frequencies in environmental hazards such as humidity, rain, snow, and wind.

Industry needs a controlled, multi-dimensional test environment to observe RF behaviors, ensure that devices receive signals clearly, and determine the number of degrees of beam steering of microwaves required. There is also a need to understand how well the device is determining direction. If minimal noise exists, determining direction is easy. In the automotive industry, direction determination would help determine how far away autonomous cars are from each other. In poor weather conditions, antennas can get wet or otherwise affected, which inhibits the ability to determine direction. To help address this problem, NIST could consider developing a test platform for these environmental conditions while accounting for different RF conditions.

Other concerns related to lack of equipment standards include determining how device performance varies in terms of peak packets received. Safety applications must be concerned with black swan events (airplane flew by very quickly) as they would affect antennas and beam steering of microwaves. Measuring range and direction accurately in cluttered environments will become increasing important to data systems using antennas. Determining range and direction requires two-way communications (two transceivers); the automotive industry is also using radar.

31. Need to re-design the control plane to improve performance (latency, throughput) and scalability.

Today's control plane protocols are heavily influenced by circuit-switched technology and other legacy environments. This makes current control plane protocols very "heavy-weight" and extremely cumbersome. Control plane protocols in the cellular context maintain 
excessive amounts of session state and involve a very large number of message exchanges to establish a communication session, handle mobility or deal with other mobile device state transitions. Because of the partitioning of functions across a number of distributed components, there are also significant inefficiencies in the protocol today. Hardware-based platforms that limit flexibility and scalability continues to be a problem.

The working group posited that fundamentally different control plane protocols need to be designed in order to support future applications and system architectures (especially things like IoT and small cell network topology). Future applications and architectures will involve a tremendous amount of messaging, even for simple functions. The community needs a more cohesive effort to understand the best way to put new control plane protocols together.

The working group discussed the emergence of software-defined networks (SDN) and the separation of the data plane from the control plane but believes that more research is needed to understand the impact of partitioning these functions. The working group also discussed the need to minimize the energy consumption of the device and make it more time-efficient; it is not clear that separating control plane functions is the best way to do this.

One suggestion that was discussed was the idea of adaptive Layer 2 and Layer 3 protocols that are smarter and more efficient, resulting in low overhead and high throughput. Adaptable protocols will help accommodate an increasing number of devices in multi-hop environments.

32. Need to understand how AI controls methods such as machine learning and deep neural networks will behave, be architected, and be measured.

Today's AI control methods require the central decider to have the same data as the other agents in the system(s) to make consistent conclusions - this results in extreme amounts of data transfer and a massive data crunch.

Efforts are underway today to reduce the amount of data being transmitted to a central decider and required for AI but no effective solution exists yet. Distributed architecture does not solve this problem. There a lot of distributed components in the control plane, including base station, device, mobility management, and all need to come to a consensus before running. This takes too much time. Separating the control and data plane helps somewhat, but there are still inconsistencies and need additional messaging before getting consensus / data flow.

33. Need ability to measure and demonstrate the effectiveness of architectures.

Today's network architecture effectiveness is measured and demonstrated by data throughput, control transaction throughput, and latency. These measures do not consider the interaction between the data and control planes. Control transactions may often block the processing of data. When there is interference between the two planes, it impacts throughput substantially. This needs to be more effectively measured and researchers need to be more aware of this.

34. Need continued improvement in RF front ends for hardware 
The working group discussed how RF behavior is becoming increasingly digitized but it is not understood how that will impact network architecture or waveform design. If waveforms could be rapidly created by a future programming language, how will devices be able to run those programmed waveforms? The ability to digitize data (similar to weather mapping) and the ability to determine how much filtering can be digitized are two gaps that exist in this area today.

Antenna design was also discussed as a gap related to architecture technology. Developing new analog antennas is difficult and costly. There is a huge engineering gap in ability to rapidly develop cost-effective mobile antenna array.

35. Need to improve beam forming techniques since it will become increasingly important into the future.

Future higher-frequency millimeter systems will rely on the antenna system to not only provide directional selectivity and improve strengths of weak signals but also put increased importance on accuracy of the imaging properties of the directional data such as direction and range. The working group discussed how beamforming to find the strongest signal works well and that the auto industry is trying to determine the direction of the waveform. The point is to connect information with safety, which makes the exact direction of a beam very important. Knowing the direction of where beams are coming from can help prevent hacks (by rejecting beams from a certain direction) and may exclude based on that information (exact angle, lack of errors). This is especially important for applications in extreme weather conditions. Many systems do not currently know when they are not performing to standard.

36. Need to develop software mechanisms that enable continued, robust, effective operations by pieces of the system when (1) unexpected conditions arise, either in the environment or in the system, or (2) connectivity to the core is impaired.

The working group discussed that future generation wireless networks will be more complex from a resiliency perspective and that there is a need to look for complex mechanisms that enable continued operation under all unexpected scenarios and conditions. Devices will come on and go off the network and we do not have a great way to manage this. Networks that are software based may help the management of this, but should only introduce what is essential. The working group argued that the in future generation wireless network architectures, the core will not be as relevant; there will still be centralized areas where one can collect information, but the core may only provide vague guidance. Edge devices will need to be more adaptable.

37. Need to develop new protocols that support mesh networking.

The working discussed how future generation networks will need the ability to connect millions of devices on the edge, potentially necessitating a move away from probabilistic to determination-based communications. Working group members also discussed how difficult it is to ensure deterministic communications, but also having probabilistic communications as a backup is very useful. Working group members mentioned that WiFi, IEEE Std. 802.11F, could be used as an example when exploring this topic. 
38. Lack of a management and operations architecture for a world of tunable devices and devices operating in millimeter bands.

The working group discussed how devices operating in the mmWave radio band frequencies are harder to communicate with because operating distances are shorter; distributed controls and instructions need to be developed to address this as well as mechanisms that ensure mesh networks behave in the desired fashion as they become increasingly dynamic.

39. Need to manage the accuracy and qualities of antenna performance beyond gain and signal rejection.

The working group argued that managing the qualities of antenna performance will be increasingly important as antenna systems are utilized in real-time automotive and drone safety systems.

\section{B.6. Protocols (November 4, 2016)}

The Future Generation Wireless R\&D working group has defined the Protocols vertical as "Methods for organizing the information carrying capacity of an underlying medium in order to accomplish communications tasks." For example, the working group cited the need to develop more straightforward techniques to measure, model, and simulate network protocol performance as a critical enabler for future mission critical wireless applications that require highly confident estimates of latency, throughput or other performance metrics.

The measurement, technology, and management gaps detailed below represent a summary of working group discussions in November 2016.

40. Need to improve the security, simplicity and accuracy of measuring performance of today's protocols.

The working group argued that one of the key evaluation challenges facing industry is the critical need to develop more simple, straightforward ways to measure network protocol performance given the mission critical nature of future generation wireless application requirements. For example, future generation wireless application areas such as remote surgery or autonomous vehicle operation will require minimal latency. Since a single point of failure in these future services would present significant risk to the safety of both users and operators of these applications, industry needs to develop protocols that can more accurately and confidently test requirements such as latency before these applications will be adopted in widespread fashion.

One participant described the lack of "simplicity" of measuring protocol performance today either in terms of implementation complexity or some more abstract measure (e.g., state diagram). For example some transport protocols, performance measured in fraction of available bandwidth occupied (efficiency) is relevant, but this is not a metric for control and signaling protocols. In other cases, delay, measured in round-trip times, may be a concern, particularly if negotiation is required.

Several participants conceded that much of industry is simply not aware of what barometers are being used to measure protocol performance today. With this in mind industry needs to establish more commonly understood techniques to measure latency, total throughput, aggregate throughput in a multi-user environment. There may be accurate ways to measure 
protocol performance today, but working group participants were not able to identify any agreed upon techniques used across industry stakeholders today.

$5 \mathrm{G}$ 's target for reduced latency for virtual reality requires users to stream video, large files, and applications. Latency will matter greatly in these future applications, and it will be up to industry to design protocols that can meet these targets. Latency will also matter greatly in vehicle-to-vehicle (V2V) communications and telemedicine. Working group participants agreed that some of the most highly publicized application areas for $5 \mathrm{G}$ and beyond will need protocols to enable much more responsive communications loops than what is available today.

However, the working group provided several examples of current R\&D projects that are working to address the need for new protocols. C-test.net is a measurement of latency, but industry is not aware of how reliable or effective this will be for future generation applications. The working group also cited AMIMON - an Israeli provider of high definition video links for unmanned aerial vehicles (UAVs) - as another example of an organization developing proprietary latency protocols that could serve as a model for reducing the round trip time of communications significantly. Although as these examples might suggest, proprietary protocols for meeting stricter latency requirements exist today. Although it may not be difficult to design these new protocols from a technology standpoint - and that digital measures for measuring latency could exist - new protocols will need to be standardized. For example, industry will need to establish whether latency needs to be measured by net or gross and what to consider as the beginning or end of the measurement. The working group argued that standardizing future protocols represents a more significant gap than the development of the protocol itself.

In addition to latency protocol standardization, the working group identified the need for standardized, well-defined throughput protocols as a key measurement gap going forward. When defining throughput protocols industry will need to specifically consider multi-user environments. Many participants agreed that definitions for latency need to be clarified, and that industry needs to better understand reduced latency targets.

Finally, the working group noted that measurement standards developed for MIMO testing will not be appropriate for mmWave, but performance metrics must be defined for these areas and other key enablers of future wireless generation capabilities. In order to achieve this, the working group recommended that standards bodies extrapolate existing standards development activities and apply them to define performance requirements and how to measure or compare the performance of emerging technologies such as massive MIMO and mmWave.

41. Need to improve techniques for modeling and simulation of future network protocols

In addition to more clearly defining protocol standards and measurement criteria, the working group indicated that current modeling and simulation techniques will not be suitable for future network protocols. Participants used several application areas within the vehicleto-vehicle communications space as examples of industries pursuing new simulation methods. Specifically the working group cited advanced driver assistance, collision avoidance and autonomous vehicle operation as areas in which 3GPP is pursuing new simulation techniques. For example, vehicular communications researchers are investigating 
how to touch the edge of the network to reduce latency and how deep packets need to go into the core network to maintain network performance from a data-rate perspective while minimizing latency. The challenge of developing protocols that balance the tradeoff between low latency and high data rate throughput is a critical area of need if emerging applications such as autonomous vehicle operation are to be widely adopted in the foreseeable future. Ideally, industry will improve its ability to do direct vehicle to vehicle communications without a network, while touching the network eNodeB and not go any farther than necessary into the core network. This area needs more R\&D attention.

The working group also pointed out that the sheer amount of protocols today makes it extremely complex and cumbersome to model network performance, and that this complexity will only increase in the future. If protocol simulation techniques do not undergo fundamental innovation, researchers may require multi-processors, neural networks, and supercomputers to handle the computing power needed to run future generation protocol simulation. The working group also indicated that it would be very difficult to validate the accuracy of protocol models if new approaches are not developed. The working group discussed in detail whether industry will develop new tools or approaches or will simply try to extend what is used today to its logical limit.

During this discussion participants argued that models will likely use the same parameters but the density of inputs would grow significantly due to the fact that industry can safely assume more users in a given area with higher data rates and higher demand for services. Researchers are less able to isolate features today than they were in the past. Because it will not be possible to solve individual issues at the protocol level, the research community will need to shift its mindset to begin considering various model components together (especially for beamforming and tracking).

The working group anticipates that existing simulation tools will continue to improve, but that simulation tools for protocols have narrowed recently. This means that industry and academia need to develop their own platforms for modeling and simulation tools. As model complexity increases so does the computational power required to run these models, making improving computational power and efficiency a critical gap for research organizations to address in the short term. Specifically, the accuracy models for future applications (i.e., V2V) needs to be improved and new beamforming techniques will need to be developed. In short, the working group believes that modeling complexity will increase and that complexity is quickly catching up with existing tools. As a result, industry and academia need to think of ways to innovate fundamental modeling and simulation tools that can accommodate protocols that will exist in 10 years to 15 years. With denser networks hosting more users with higher data rate demands, the community will need to evaluate all aspects of future networks in a closed system loop.

42. Need to develop new protocols that can recognize and allocate resources based on asymmetric uplink vs. downlink data demands.

Industry needs to look at how protocols will allocate different amounts of data to different users with multiple devices that each have differing data demands. Developing new protocols that can deal with asymmetry -- varying "Uplink vs. Downlink" data demand was cited as an important research area going forward. For example, future WiFi networks that are data- 
centric that enable asymmetric loading will need to perform well in environments where different users are loading the system differently. Emerging applications such as Periscope which enables people to video blog live while sending or receiving messages - uplinks very heavily while only receiving occasional updates. This application has much higher upstreaming than down streaming and calls out the need for protocols that can navigate dynamic data usage patterns seamlessly. Other applications may be asymmetric in the other direction, and industry need protocols that can handle this non-uniform distribution of data and user demands.

43. Protocols need to be increasingly implemented directly in hardware to accommodate higher data rates.

The working group recognized that the telecommunications industry will need to navigate a tradeoff between the computability of software defined networks and higher data rates. This will necessitate more flexible integration of hardware and software defined networks so that many protocol features, including field-programmable gate arrays (FPGAs), will be implemented in hardware. While increasing data rates will require greater protocol integration with hardware, some features such as cryptographic functions or the lowest-level protocols in high performance specialty applications such as Tb routers may not be deployed directly in hardware.

44. Need to standardize Protocols for Air Interfaces, Networking, and IP Networks.

The working group indicated that researchers today are developing a variety of Open Source Network Protocols, but these activities are fragmented and being done in isolation. The working group also sees a need to develop standards related to Internet protocols. One specific area of need is the lack of an extensible wireless data standard (i.e., general eXtensible Markup Language (XML) or JavaScript Object Notation (JSON)). The communications industry has also struggled for many years to develop and deploy a sufficiently secure architecture for IP. Securing names and identity on the Internet is widely seen as a fundamental problem, and the working group recognized an opportunity to determine better ways to secure identifiers at all open systems interconnection (OSI) layers. However some working group participants did not see this as an actionable challenge to take on as a result of this report.

45. Need to establish new protocols for picocells in order to catalyze network densification.

46. Need to develop new protocols for spectrum sharing.

47. Need to develop reference implementations of protocols (e.g., for interoperability testing, teaching, and modeling) to serve as building blocks for real systems.

48. Managing protocol configurations, including changes in live systems, and state in ways that are simpler and more flexible than classical simple network management protocol (SNMP) techniques. 